\title{
RESULTADOS PRELIMINARES EN LOS ESTUDIOS DE EFICIENCIA ENERGÉTICA DENTRO DEL PROYECTO I+D+i OPTIREG: GESTIÓN EFICIENTE DE REGADÍOS
}

\author{
Naranjo Hernández, $D^{1}$., Colom Delgado, $S^{2}$., Blanco Redondo, $J C^{3}$., González Muñoz $L G^{4}$., \\ Iglesias Gómez, $S^{5}$. \\ ${ }^{1}$ Responsable técnico de proyecto OPTIREG1316, Gerencia de Arquitectura e Ingeniería \\ (Tragsatec), Julián Camarillo 6, 28037 Madrid, dnh@tragsa.es \\ ${ }^{2}$ Responsable técnico de proyecto OPTIREG1316, Gerencia de Arquitectura e Ingeniería \\ (Tragsatec), Cobalto 3, 47012 Valladolid, scd@tragsa.es \\ ${ }^{3}$ Responsable técnico de proyecto OPTIREG1316, Gerencia de Arquitectura e Ingeniería \\ (Tragsatec), Cobalto 3, 47012 Valladolid, jblanco6@tragsa.es \\ ${ }^{4}$ Responsable técnico de proyecto OPTIREG1316, Departamento de Obras y Servicios \\ (Tragsa), Cruz Roja de León 26, 24008 León, Igonzal2@tragsa.es \\ ${ }^{5}$ Responsable de apoyo y supervisión de proyecto OPTIREG1316. Subdirección de I+D+i \\ (Tragsa), Conde Peñalver 84, Madrid, siglesia@tragsa.es
}

\section{Resumen}

El grupo de trabajo WP3 "Eficiencia energética" en el marco del proyecto I+D+i OPTIREG "Gestión Eficiente de Regadíos" del grupo Tragsa, lleva a cabo diversas actuaciones encaminadas a conocer el estado energético y organizativo de la gestión de las CC.RR. modernizadas, buscando metodologías que mejoren las herramientas de diagnóstico de la situación hídrico-energética de una C.RR., como también estrategias operativas que mejoren la eficiencia energética adaptándose a la idiosincrasia y condición de cada caso particular. Para alcanzar estos objetivos, los trabajos se están centrando en varios aspectos:

- Recopilación de medidas efectivas de eficiencia energética aplicadas a los diversos ámbitos de la infraestructura y organización en que se diversifica una C.RR.

- Caracterización de CC.RR. de Castilla y León con indicadores conocidos y nuevas propuestas de los mismos (benchmarking).

- Evaluación de los rendimientos de la EB mediante el estudio de las curvas globales $\mathrm{H}-\mathrm{Q}$, P-Q y Rto-Q.

- Estudio de curvas de frecuencia de caudales.

- Elaboración y calibración de modelos hidráulicos de EB y red de riego en dos zonas piloto para análisis de escenarios simulados con fines a valorar nuevas estrategias de gestión que mejoren la eficiencia energética sin que se vea mermada la calidad del servicio (presión y caudal en el hidrante), ni aumenten el coste energético anual.

- Pasarela de comunicación entre el programa de gestión de riegos Progar y Telegestar.

\section{1- Introducción}

Las políticas tanto de nuevos regadíos como de modernizaciones y consolidaciones de los ya existentes llevadas a cabo en las últimas décadas a través de diversas actuaciones de ámbito tanto estatal como autonómico (Plan Nacional de Regadíos, Plan de Choque, Estrategia para la Modernización Sostenible de los Regadíos, Planes de Cuenca,...) si bien están afrontando con éxito el reto de optimizar el uso del agua (eficiencia hídrica), en el sentido de disminuir los volúmenes transportados, servidos y consumidos (objetivo preferente de los planes y programas ejecutados, entre otros beneficios asociados como son 
la vertebración del territorio y la dinamización el sector agrario,) por medio de la implantación de sistemas de riego más eficientes en su manejo y aplicación del recurso y de la mejora de las infraestructuras de transporte y distribución de agua, llevan asociados una alta demanda energética al pasar del riego tradicional por superficie (canteros, inundación, surcos...) suministrado por un sistema de canales y acequias que trabajan con un flujo en lámina libre a un riego a presión (aspersión y goteo principalmente) abastecido por una red de distribución colectiva de tuberías presurizadas enterradas, sistemas que requieren de importantes cantidades de energía eléctrica para su funcionamiento cuando por condiciones de la topografía del terreno (cotas de las zonas de consumo mayor respecto a la cota del punto de captación) o por el origen del agua de riego (subterráneo y/o superficial) no es posible realizar un aprovechamiento racional de la energía gravitatoria disponible en la naturaleza y es necesario recurrir a grupos de bombeo.

En una visión global, se constata en muchos regadíos una alta dependencia energética cuyo coste asociado presenta una tendencia al crecimiento debido a escenarios de uso de las infraestructuras y adopción de esquemas de operación distintos o alejados a los criterios de diseño y/o manejo de proyecto en que la rentabilidad económica quedaba justificada: cambios de las superficies regadas o del sistema de riego en parcela con otras necesidades de presión/caudal y requerimientos energéticos; modificación de los cultivos de la alternativa con otras exigencias hídricas; reforma del sistema de tarifas eléctricas con distintas franjas horarias; deficiencias arrastradas de un dimensionamiento hidráulico inadecuado de las instalaciones (por infra o sobredimensionado) o en el que se ha dado una concepción pobre de la estructura de la red; deficiente o incompleta gestión que tenga en cuenta el binomio agua-energía tanto en su uso como en su contratación; envejecimiento prematuro de las instalaciones por un mantenimiento insuficiente; funcionamiento de los equipos de bombeo alejados de su rendimiento máximo; y un aumento progresivo de los precios de las tarifas eléctricas. Es decir, que en conjunto, se esté operando en puntos alejados de las previsiones iniciales, pudiendo dejar en entredicho el tecnificado regadío español.

Además, la mayoría de los grandes proyectos de intervención pública han venido acompañados de la incorporación de una abundante tecnología, automatismos e instrumentación (telecontrol, sistemas SCADAs, sensores, PLCs, variadores de frecuencia, arrancadores electrónicos, sistemas redundantes de vigilancia y control, programas informáticos específicos para la gestión de los riegos...) al objeto de facilitar el manejo de la infraestructura y la gestión del agua, quedando a veces obsoleta, relegada al abandono o infrautilizada debido a una falta de mantenimiento, a la complejidad y dificultada de su implantación y uso, a una nula o escasa preparación técnica del personal que tiene que manejarla, al limitado seguimiento de los Organismos públicos que han promovido las obras, o a un uso ineficiente o incompleto con respecto a las posibilidades con las que están dotadas, si bien es cierto que hay muchas Comunidades de Regantes (en adelante CC.RR.) que han sabido adaptarse al cambio y sí que están gestionando y manejando sus instalaciones de una forma racional y eficiente, alcanzando niveles óptimos de control con los avances tecnológicos incorporados, ya sea con personal propio o a través de servicios contratados a terceros.

En lo que respecta a las operaciones de explotación de todo el sistema (red de riego, estación de bombeo - en adelante EB -, balsas, filtros generales, edificaciones, arquetas...) entendida como el conjunto de tareas, procesos y operaciones interdependientes entre sí más o menos complejos: peticiones y ejecución del riego, control del consumo de agua, contratación de la potencia eléctrica, restricciones horarias de uso de los equipos de bombeo, garantizar con calidad el servicio (principalmente asegurar la presión y caudal requerido en los puntos de consumo), facturación, mantenimiento, manejo de situaciones no previstas como restricciones de agua por sequías o interrupciones temporales del servicio por fallos...se verifica en algunos casos dificultades o ineficacias en las operaciones de 
gestión hídrica/energética o que éstas no son realizadas de forma eficiente, correctamente o de forma completa con respecto al aprovechamiento potencial con que podrían llevarse a cabo. Además, se confirma que en algunas instalaciones en las que existe la posibilidad de obtener registros históricos de parámetros hidráulicos y energéticos, no se efectúa un análisis o un seguimiento de éstos con fines a conocer el comportamiento real de la instalación con vistas a parametrizar la gestión, el funcionamiento de los equipos, detectar disfuncionalidades 0 proponer ajustes 0 formas más eficientes y óptimas de gestión/operación/regulación.

Finalmente, se contrasta que existe una amplia variabilidad de tipología de CC.RR. por diferentes motivos (sistemas de riego en parcela, topografía de la zona, procedencia del agua, cultivos implantados, costumbres, uso de la tecnología implantada, organización y tamaño de la propiedad...), por lo que todo intento de modelizar un sistema de gestión único (enfocado a mejorar la eficiencia energética e hídrica) y para todos las tipologías de CC.RR. estará condenado al fracaso.

Con todo, las posibilidades de mejorar la eficiencia energética e hídrica desde varias estrategias, y así atenuar los costes energéticos asociados, tanto en regadíos que poseen alta tecnología como en aquellos más precarios y según las tipologías variadas de CC.RR. son muy amplias.

\section{2- Objetivos del proyecto}

El Grupo Tragsa, como conocedor del sector por su larga trayectoria tanto por las obras de regadío que lleva ejecutando en todo el territorio nacional como por su labor en la redacción de proyectos y por las encomiendas de gestión y/o mantenimiento de varias zonas regables, consciente de la necesidad de analizar y mejorar los modelos actuales de gestión y explotación de las infraestructuras de regadío de una forma óptima y sostenible de los recursos, en especial de la energía, apuesta por dar soluciones ante las diversas realidades existentes en el regadío a través de la aplicación e incorporación de innovaciones tecnológicas y de investigación mediante el desarrollo del proyecto I+D+i "Gestión Eficiente de Regadíos" (OPTIREG 1316).

Para lograr sistemas de gestión responsables que integre todos los recursos (hídricos, energéticos, tecnológicos, medios humanos), adaptativo a las particularidades de las CC.RR. y que busque su eficiencia en términos económicos, sociales y ambientales, con disposición de tomar decisiones de gestión y/o realizar propuestas de mejora viables desde el punto de vista práctico en base a: la capacidad técnica disponible (manejo de software para simulación y análisis de modelos de redes y EB, uso de las TIC, instalación de sensores para registro de datos, energías alternativas...); a las posibilidades que ofrece el mercado eléctrico (compra directa de energía a través del pool); y a la facultad de autoevaluación de la gestión realizada mediante el seguimiento y análisis del uso conjunto del agua y de la energía en base a parámetros e indicadores de gestión con el objetivo de detectar posibles ineficiencias y minimizar el consumo energético, al objeto de lograr una rentabilidad y sostenibilidad de aquellas infraestructuras de regadío que dependen de la energía eléctrica para su funcionamiento, se concibe OPTIREG.

El proyecto OPTIREG trabaja en una serie de bloques programados $e$ interrelacionados entre ellos, que son: implantación de energías renovables, mercados eléctricos y compra-venta de energía, eficiencia hídrica y eficiencia energética, orientadas al objetivo principal del mismo, que es optimizar la eficiencia hídrica y energética y la tecnificación de las zonas regables que permita su viabilidad económica.

Para el adecuado desarrollo del proyecto, se están constituyendo zonas piloto donde se recaba información y se aplicará la incorporación de soluciones técnicas de asesoramiento al regante que permitirá un Know how extrapolable a nivel nacional. 
Dentro del bloque de trabajo WP3 "Eficiencia energética", el objetivo principal del proyecto es obtener una visión global de la tecnología aplicada en la gestión de las CC.RR. como consumidores de energía, detectando sus disfuncionalidades para aplicar diversas estrategias prácticas orientadas a mejorar la eficiencia energética haciendo uso de la tecnología disponible. Para ello la metodología de trabajo del bloque WP3 consiste en realizar actuaciones paralelas con contenidos temáticos de gran diversidad que a continuación resumimos:

- Actualización y conocimiento del estado del arte.

- Recopilación de medidas de eficiencia energética.

- Caracterización de CC.RR. de Castilla y León (en adelante CyL).

- Obtención de datos hidráulicos y eléctricos en zonas piloto y CC.RR. colaboradoras. Análisis y tratamiento de los mismos. Elaboración de curvas, parámetros e índices para mejorar la gestión y regulación con fines a mejorar la eficiencia energética.

- Auditorías energéticas en las zonas piloto siguiendo la metodología del IDAE.

- Elaboración y calibración de modelos hidráulicos de EB y red de riego de las zonas piloto.

- Análisis de escenarios simulados con fines a valorar nuevas estrategias de gestión que mejoren la eficiencia energética sin que se vea mermada la calidad del servicio (presión y caudal en el hidrante), ni aumenten el coste energético anual. Comparativa entre escenarios simulados y reales.

- Pasarela de comunicación entre el programa de gestión de riegos Progar y Telegestar. Protocolos operativos de funcionalidades disponibles en Telegestar (supervisa, valida programación, organiza riegos, optimización bombeos, optimización regulación de bombeo) en las zonas piloto.

En el presente trabajo se presenta los primeros resultados del grupo WP3 obtenidos de las principales líneas de trabajo en las que se están desarrollando diversas actividades orientadas a analizar el funcionamiento de varias instalaciones colectivas de riego y adoptar estrategias que mejore la gestión de la red optimización el uso de la energía.

\section{3- Material y descripción del proceso de trabajo}

La efectividad de los estudios, conclusiones y medidas de mejora que pretende buscar y obtener el proyecto OPTIREG se está logrando gracias a la colaboración de varias CC.RR., a las que se les han realizado visitas para conocer su actual situación, organización del riego, nivel de automatización, estado de los equipos y su interés de colaboración como zonas piloto. De momento, se han realizado entrevistas a seis CC.RR. de CyL, de las cuales se han firmado convenios de colaboración con cinco de ellas, a la espera de efectuar otras visitas pendientes y que se sumen otros convenios al proyecto.

Con las CC.RR. que se han firmado convenios de colaboración, los estudios que se están realizando son en base a los datos recogidos en la memoria del SCADA, como también los proporcionados en las facturas eléctricas y en las curvas de potencia consumida facilitadas por la empresa distribuidora eléctrica. En base a esta información, los estudios y análisis principalmente están orientados a obtener las curvas de frecuencia de caudales y parametrizar las curvas operativas de la EB altura/caudal $(\mathrm{H}-\mathrm{Q})$, potencia/caudal $(\mathrm{P}-\mathrm{Q})$ y rendimiento/caudal (Rto-Q) para evaluar en qué rangos de eficiencia se está bombeando los caudales demandados y proponer medidas de mejora.

Se pretende en un próximo estudio realizar una evaluación individual de cada una de las bombas que compone la EB mediante la instalación de un analizador de redes y un caudalímetro portátil de cara a determinar las prestaciones y el rendimiento de los equipos individuales y en su conjunto, y poder conocer: los desvíos existentes entre las curvas emitidas por el fabricante (ya sean de catálogo o de banco de ensayo) y las curvas reales; detectar si alguna bomba puede estar trabajando con rendimientos menores al esperado por 
envejecimiento, incorrecta elección o mala regulación; y reconfigurar la secuencia de activación/parada de la composición de los grupos (fijos y velocidad variable) que mejore la curva global Rto-Q de la EB seleccionando los puntos de transición de estado.

Por otro lado, se están llevando a cabo estudios pormenorizados de eficiencia energética en dos zonas pilotos en las que el Grupo Tragsa tiene encomiendas de gestión y mantenimiento desde hace varios años. Son las CC.RR. del Canal de la Margen Izquierda del Porma (en adelante CRRMIP) y del Canal del Páramo Bajo (en adelante CRRCPB), ubicadas ambas en la provincia de León. En general se trata de dos grandes zonas regables con redes presurizadas mediante bombeos de inyección directa con riego a la demanda programada, que cuentan con hidrantes de riego automatizados con telecontrol.

En ambas zonas piloto se dispone de muchos registros de parámetros de uso de las redes, principalmente tiempos y caudal de cada riego por hidrante, horario de apertura y cierre de los mismos, presiones en varios nodos, así como también de los que acontecen en la EB ( $k W h$ consumidos, presiones y caudales bombeados...) para cada escenario de riego que se tenga programado. Como también se dispone de la información apropiada para modelizar la red y la EB mediante herramienta informática apropiada, se están llevando a cabo estudios hidráulicos-energéticos enfocados a proponer medidas de gestión de mejora de la eficiencia energética e hidráulica cuantificando el efecto de su implantación para estudiar su viabilidad. Estos estudios de mayor entidad en los que se hace necesario el modelo hidráulico de la red (y su calibración) también se llevarán a cabo en varias de las CC.RR. colaboradoras según la situación energética en que se encuentre y la prioridad de mejora que pueda obtenerse.

Para la medida in situ de la presión en aquellos puntos de la red que sea de interés el equipo WP3 cuenta con instrumentación de campo que ha adquirido como son dos sondas portátiles (modelo CPH6200 de WIKA) con registro continuo y alta capacidad de almacenamiento, así como 10 transductores de presión.

Además de las zonas regables, ya sean pilotos o colaboradoras, se han firmado dos convenios más de colaboración: uno con el equipo de GESTAR de la Universidad de Zaragoza con el fin de implementar la herramienta Telegestar en combinación con el programa de gestión de riegos PROGAR en las zonas piloto de León; y otro con SEIASA Delegación Territorial Norte por el cual han facilitado los informes de final campaña de 30 CC.RR. de un período de dos ó tres años al objeto de realizar un análisis de parámetros e índices de gestión para caracterizarlos con metodología de benchmarking.

\section{4- Recopilatorio de medidas de eficiencia energética}

Una de las primeras actividades que se llevó a cabo dentro del proyecto OPTIREG fue la recopilación de documentación técnica específica relacionada con los objetivos del equipo WP3 para tener una visión global del estado actual del arte relacionada con la gestión y la eficiencia energética. La documentación recopilada proviene de cursos, revistas especializadas, simposios, congresos, encuentros formativos, jornadas técnicas, tesis doctorales, IDAE, etc. Si bien mucha de esta documentación menciona, cataloga o incluso explaya medidas de eficiencia energética en CC.RR., bien es cierto que no se encontró un documento que aglutinara todo el abanico de posibles medidas de eficiencia energética que se encuentran dispersas en los distintos trabajos de investigación analizados.

Con ello, una de las actividades del proyecto OPTIREG ha consistido en disponer en un documento único la recopilación de toda una batería de medidas y acciones orientadas al ahorro y eficiencia energética en el regadío (principalmente para reducir la factura eléctrica y el consumo de energía total en la campaña) si se aplican de manera adecuada. No pretende ser un catálogo exhaustivo, sino una exposición de todas las medidas que se ha ido encontrando en la distinta bibliografía, así como las que son producto de la propia 
experiencia que se está adquiriendo en base al proyecto OPTIREG.

El coleccionable de medidas de eficiencia tiene interés y practicidad a todos los usuarios y principales actores de la explotación de la instalación (gestores, regantes, guardas, operarios, administrativos...) que tienen su parte de responsabilidad en la eficiencia energética final del mismo. Algunas de las medidas precisan de esfuerzos económicos, de investigación y de innovación para que pueda ser puesta en marcha en aplicaciones a gran escala, pero en numerosas ocasiones será rentable con nulo o bajo coste de implementación, consiguiendo significativos ahorros tanto de potencia como de energía sin comprometer la calidad del servicio,

El trabajo recopilatorio de medidas se ha dividido en varios bloques interdependientes entre sí, pero con suficiente estructura individual en cada uno de ellos: equipos de bombeo (11 medidas generales y 15 particulares); regulación de la EB (15 medidas generales y 13 particulares); equipamiento instalaciones colectivas (5 medidas generales y 13 particulares); equipamiento en instalaciones de parcela ( 11 medidas generales y 40 particulares); consumo de agua (13 medidas generales y 19 particulares); operaciones de gestión (11 medidas generales y 8 particulares); contratación del suministro eléctrico (14 medidas generales); formación y concienciación (11 medidas generales); y toma de datos y mediciones.

Además de estas medidas de eficiencia energética, se está trabajando en otra línea paralela a este fin y orientado al diseño técnico de nuevas infraestructuras de riego colectivo utilizando criterios de eficiencia energética (proceso de reingeniería).

\section{5- Caracterización de CC.RR. de Castilla y León}

Una parte del trabajo que lleva a cabo el grupo WP3 consiste en analizar la relación agua y energía en varias zonas regables modernizadas, principalmente de CyL. El objetivo de esta parte del proyecto OPTIREG es obtener indicadores que permitan evaluar la situación energética e hídrica (ratios de consumo y de costes de agua y energía) de las CC.RR. estudiadas, y cuya cuantificación sean útiles para la formulación de recomendaciones que permitan racionalizar su uso, obtener valores energéticos de referencia del regadío castellanoleonés, comparar los valores entre la misma Comunidad de Regantes (en adelante C.RR.) y entre varias CC.RR. (benchmarking) y proponer nuevos índices de estudio.

El abanico disponible de CC.RR. a evaluar es de 30 (datos facilitados por SEIASA Delegación Territorial Norte), y en esta ponencia se presenta los resultados obtenidos en 12 de ellas durante un periodo de dos años (2010-2011), aunque en alguna C.RR. tiene datos hasta un periodo de 5 años.

Las 12 CC.RR. seleccionadas poseen unas características comunes:

- Cultivos: predominan tanto cultivos herbáceos de invierno (trigo, cebada, centeno, avena y colza) a los que se les aplica riegos en sus últimas fases de desarrollo, como los de verano, siendo éstos los que se siembran en mayor proporción (maíz principalmente, seguido de remolacha y patata). Destaca en menor medida alfalfas y pratenses, girasol y hortícolas. En cultivos anuales, no se realiza una segunda cosecha, con alguna excepción. Los cultivos leñosos tienen una presencia escasa, predomina la viña para vinificación, así como el cultivo del chopo y algún frutal (manzanos y perales).

- Sistemas de riego: la mayoría de las CC.RR. disponen de riego por aspersión en sus varias vertientes (coberturas, pivots y cañones), si bien en una gran parte de la superficie el riego se realiza con cobertura aérea, y en menor medida con el resto de los sistemas. El riego por goteo apenas es presencial, excepto una C.RR. que tiene bastante proporción de este sistema por la implantación de la viña.

- Procedencia del agua: en todas ellas procede de captaciones superficiales, ya sean de tomas directas de ríos o de embalses de regulación, transportando el agua por medio de 
un sistema de grandes canales de riego hasta cabecera de la zona regable. No hay presencia de pozos ni de recursos hídricos subterráneos.

- Calendario de la campaña de riegos: si la climatología viene muy desfavorable (calurosa), los primeros riegos se suelen dar a mediados de marzo, pero por lo general en un año normal no es hasta principios-mediados de abril cuando se empieza a regar. Finaliza la campaña entre septiembre y principios de octubre.

- Condiciones climatológicas durante la campaña (abril-septiembre): la precipitación acumulada promedio es de $157 \mathrm{~mm}$, la $E_{\text {o }}$ acumulada promedio es de $844 \mathrm{~mm}$ y la velocidad media del viento es de $1,75 \mathrm{~m} / \mathrm{s}$, por lo que los sistema de riego (mayoritariamente aspersión) no se ven muy influenciados en el proceso de aplicación del agua por este factor negativo poco controlable.

- Sistema colectivo: en general se trata de CC.RR. que han tenido procesos de modernización recientemente, en el que predomina la misma tipología de infraestructura que consiste en una red colectiva de tuberías a presión de topología ramificada que finaliza en un hidrante general compartido (multiusuario) entre varios regantes, y a partir de éste se llega a la parcela mediante un sistema de tomas individuales, aunque existe variantes tanto de la composición del hidrante y de la toma (valvulería) como en el sistema de llegar hasta la finca. No existen rebombeos individuales en las fincas.

- Estaciones de bombeo: todas las CC.RR. poseen una o varias EB si hay sectores independientes. Todas las EB se abastecen de energía eléctrica en línea de media tensión y con contratación de tarifa eléctrica de seis periodos (potencia mayor de 450 $\mathrm{kW}$ ). Los equipos de elevación son a balsa para riego por gravedad desde ésta o de inyección directa a red a una presión constante o variable según los casos.

En cuanto a los indicadores de benchmarking utilizados, se ha basado el estudio en la propuesta del "International Program for Technology and Research on Irrigation and Drainage" IPTRID (Malano y Burton, 2001), considerando también otros indicadores propuestos por diversos autores (Rodríguez, 2003; Córcoles, 2009; Tarjuelo et al. 2010; Abadía et al., 2010, Soto et al., 2014). Además de comparar los resultados obtenidos con los elaborados por los distintos grupos de investigación, se ha propuesto nuevos indicadores que no figuran en los trabajos de los autores mencionados, de cara a buscar la idoneidad de indicadores comparativos que tenga en consideración las distintas tipologías de CC.RR. con las que nos podemos encontrar. El trabajo completo analiza el valor de 58 indicadores (descriptivos, de rendimiento y de eficiencia) si bien en esta comunicación se presenta aquellos que mejor describen y permiten caracterizar las zonas regables desde un punto de vista energético. La tabla 1 describe 18 de los 58 indicadores estudiados, cuyos valores obtenidos se analizará más adelante.

Tabla 1. Descripción de los indicadores empleados (elaboración propia)

\begin{tabular}{|c|c|c|c|}
\hline ACRÓNIMO & NOMBRE DEL INDICADOR & UNIDAD & DESCRIPCIÓN \\
\hline $\mathrm{Sr}$ & Superficie regada & ha & $\begin{array}{l}\text { Superficie total de las parcelas en el sector hidráulico independiente }(\mathrm{SH}) \text { o en la } \\
\mathrm{CR} \text { que han recibido aporte de agua de riego en la campaña. }\end{array}$ \\
\hline $\mathrm{V}_{\mathrm{T}}$ & $\begin{array}{l}\text { Volumen de agua que entra } \\
\text { al sistema }\end{array}$ & $\mathrm{m}^{3}$ & $\begin{array}{l}\text { Cantidad total de agua que entra al } \mathrm{SH} \text { o a la CR, medida en los contadores } \\
\text { generales de la EB (frecuentemente son caudalímetros) } \\
\text { Incluye la cantidad de agua bombeada para riego, en redes a presión, así como el } \\
\text { agua desviada en redes por gravedad si ésta ha sido registrada en los contadores } \\
\text { generales. } \\
\text { - También incluye el agua que ha sido bombeada o derivada por gravedad para } \\
\text { realizar limpiezas de la red y la que ha fugado por roturas }\end{array}$ \\
\hline ED & Eficiencia de distribución & $\%$ & $\begin{array}{l}\text { Cociente entre el volumen de agua de riego suministrado a los usuarios y el } \\
\text { volumen de agua que entra al sistema } \\
\text { - Indica el porcentaje de agua que entra en el sistema y que no llega a los usuarios } \\
\text { finales por diferentes causas: agua fugada por fallos y roturas en la red; vaciados } \\
\text { controlados de la red por los desagües; errores de los contadores... }\end{array}$ \\
\hline VsSr & $\begin{array}{l}\text { Suministro de agua por } \\
\text { unidad de área regada }\end{array}$ & $\mathrm{m}^{3}$ & $\begin{array}{l}\text { Volumen suministrado a los usuarios del } \mathrm{SH} \text { o la CR respecto a la superficie } \\
\text { regada }\end{array}$ \\
\hline
\end{tabular}




\begin{tabular}{|c|c|c|c|}
\hline ACRÓNIMO & NOMBRE DEL INDICADOR & UNIDAD & DESCRIPCIÓN \\
\hline ICE & Índice de carga energética & mca & $\begin{array}{l}\text { - Presión media ponderada de bombeo durante la campaña de riegos } \\
\text { - Tanto en bombeos a punto fijo como en inyección directa a red representa la } \\
\text { presión media a la que han bombeado los equipos a lo largo de toda la campaña } \\
\text { como diferencia entre la presión en la impulsión y la aspiración. } \\
\text { - Si no se ha medido y promediado, la presión se corresponde con la presión } \\
\text { nominal de los equipos de bombeo especificadas en la placa de características } \\
\text { técnicas }\end{array}$ \\
\hline $\mathrm{NcSr}$ & $\begin{array}{l}\text { Potencia contratada por } \\
\text { unidad de área regada }\end{array}$ & $\mathrm{kW} \mathrm{ha}^{-1}$ & $\begin{array}{l}\text {. Cociente entre la máxima potencia contratada y la superficie regada del } \mathrm{SH} \text { o la } \\
\mathrm{CR}\end{array}$ \\
\hline $\mathrm{NcVs}$ & $\begin{array}{l}\text { Potencia contratada por } \\
\text { volumen de agua } \\
\text { suministrado } \\
\end{array}$ & $\mathrm{kW} \mathrm{hm}^{-3}$ & $\begin{array}{l}\text { Cociente entre la máxima potencia contratada y el volumen de agua suministrado } \\
\text { a los usuarios }\end{array}$ \\
\hline INc & $\begin{array}{l}\text { Índice de potencia } \\
\text { contratada }\end{array}$ & $\mathrm{kW} \mathrm{hm}^{-3} \mathrm{mca}^{-1}$ & $\begin{array}{l}\text { - Cociente de la máxima potencia contratada entre el volumen de agua que entra al } \\
\text { sistema y la altura manométrica nominal }\end{array}$ \\
\hline $\mathrm{EaSr}$ & $\begin{array}{l}\text { Energía activa total } \\
\text { consumida por unidad de } \\
\text { área regada }\end{array}$ & kWh ha ${ }^{-1}$ & $\begin{array}{l}\text { - Cociente entre la energía activa total facturada en el } \mathrm{SH} \text { o en la CR y la superficie } \\
\text { regada }\end{array}$ \\
\hline $\mathrm{EaV}_{\mathrm{T}}$ & $\begin{array}{l}\text { Energía activa total } \\
\text { consumida por volumen de } \\
\text { agua que entra en el } \\
\text { sistema }\end{array}$ & $\mathrm{kWh} \mathrm{m}^{-3}$ & $\begin{array}{l}\text { - Cociente entre la energía activa total facturada en el SH o en la CR y el volumen } \\
\text { de agua que entra al sistema }\end{array}$ \\
\hline EEB & $\begin{array}{l}\text { Eficiencia energética de } \\
\text { bombeo }\end{array}$ & $\%$ & $\begin{array}{l}\text { - Cociente entre la energía hidráulica total suministrada y la energía activa total } \\
\text { consumida }\end{array}$ \\
\hline IEa & $\begin{array}{l}\text { Índice de carga de energía } \\
\text { activa consumida }\end{array}$ & $\mathrm{kWh} \mathrm{hm}^{-3} \mathrm{mca}^{-1}$ & $\begin{array}{l}\text { - Cociente de la energía activa total consumida en el } \mathrm{SH} \text { o en la } \mathrm{CR} \text { entre el } \\
\text { volumen de agua que entra al sistema y la altura manométrica nominal }\end{array}$ \\
\hline RtNc & $\begin{array}{l}\text { Rendimiento teórico medio } \\
\text { de uso potencia en julio y } \\
\text { durante el periodo tarifario } \\
\text { P6 }\end{array}$ & $\%$ & $\begin{array}{l}\text { - Cociente entre la potencia teórica media absorbida en julio y durante el periodo } \\
\text { tarifario P6 (o en valle para el suministro } 3.1 . \mathrm{A} \text { ) y la potencia contratada para el } \\
\text { periodo considerado } \\
\text { - Representa el grado de ajuste o de aprovechamiento de la potencia teórica } \\
\text { promedio de funcionamiento de la instalación con respecto a la potencia } \\
\text { contratada para el mes de julio }\end{array}$ \\
\hline GCea & $\begin{array}{l}\begin{array}{l}\text { Gasto energético por } \\
\text { energía }\end{array} \\
\end{array}$ & $\%$ & - Cociente entre el coste del término de energía y el coste energético \\
\hline GCep & $\begin{array}{l}\text { Gasto energético por } \\
\text { potencia }\end{array}$ & $\%$ & - Cociente entre el coste del término de potencia y el coste energético \\
\hline CENSr & $\begin{array}{l}\text { Coste energético por área } \\
\text { regada }\end{array}$ & $€$ ha $^{-1}$ & - Cociente entre el coste energético y la superficie regada \\
\hline CENVs & $\begin{array}{l}\text { Coste energético por } \\
\text { volumen suministrado a los } \\
\text { usuarios }\end{array}$ & $€ \mathrm{~m}^{-3}$ & $\begin{array}{l}\text { - Cociente entre el coste energético y el volumen de agua suministrado a los } \\
\text { usuarios }\end{array}$ \\
\hline ICEN & Índice de coste energético & $€ \mathrm{hm}^{-3} \mathrm{mca}^{-1}$ & $\begin{array}{l}\text { - Cociente del coste energético entre el volumen de agua que entra al sistema y la } \\
\text { altura manométrica nominal }\end{array}$ \\
\hline
\end{tabular}

INc, IEa, RtNc, GCea, GCep y ICEN son nuevos indicadores propuestos derivados de otros índices al objeto de atenuar la discrepancia existente entre distintas tipologías de CC.RR. cuando se enfrentan ante un mismo indicador. De esta manera, tres de ellos (INc, IEa y ICEN), trata de obtener un valor de referencia que aglutine el volumen que se consume y la altura manométrica a la que se bombea. Por otro lado, GCea, GCep son dos indicadores que hace referencia al porcentaje total de la factura eléctrica que se destina a energía y a potencia respectivamente.

Los valores de los 18 indicadores de las 12 CC.RR. estudiadas se muestran en la tabla 2, en donde se hace referencia a la denominación de cada C.RR. con un primer número seguido de otro número en aquellas CC.RR. formada por varios sectores; además en cada C.RR. y sector se muestra con la referencia BB si se trata de un bombeo a balsa, con BD si se trata de un bombeo directo a red y con BB-BD si se trata de una misma EB en el que hay instalados dos bombeos independientes (uno a balsa y otro directo a red) pero en el que las mediciones de los parámetros energéticos no se puede discriminar ya que comparten el mismo contador de energía.

El tratamiento que se ha realizado a los valores obtenidos de los indicadores ha sido de estadística descriptiva (medidas de posición y dispersión de la media) para conocer la variabilidad en las campañas de estudio (en la mayoría de los casos dos años) para cada una de las 12 CC.RR., como también entre las mismas. Los estadísticos manejados para analizar la tendencia central y la dispersión de los valores son la media aritmética y el coeficiente de variación (CV). 
Determinar la similitud entre CC.RR. mediante análisis multivariante Cluster y la evolución de los indicadores a lo largo del tiempo será objeto de un trabajo específico de caracterización que se publicará próximamente.

Tabla 2. Indicadores estudiados más significativos (elaboración propia)

\begin{tabular}{|c|c|c|c|c|c|c|c|c|c|c|c|c|c|c|c|c|c|c|}
\hline Denominación & $\mathrm{Sr}$ & $\mathbf{V}_{\mathbf{T}}$ & ED & VsSr & ICE & $\mathrm{NcSr}$ & NcVs & INc & $\mathrm{EaSr}$ & $\mathrm{EaV}_{T}$ & EEB & IEa & RtNc & GCea & GCep & CENSr & CENVs & ICEN \\
\hline CRR 1.1_BB & 929 & 6.279 .850 & 85 & 5.703 & 65 & 1,18 & 177 & 2,72 & 1.904 & 0,282 & 63 & 4.339 & 57 & 74 & 21 & 193 & 0,034 & 440 \\
\hline CRR 1.2_BB & 1.020 & 6.108 .661 & 91 & 5.439 & 60 & 1,18 & 198 & 3,30 & 1.725 & 0,288 & 57 & 4.800 & 38 & 77 & 23 & 166 & 0,030 & 462 \\
\hline CRR 1.3_BB & 1.194 & 7.011 .212 & 82 & 4.762 & 78 & 1,34 & 230 & 2,95 & 1.903 & 0,326 & 65 & 4.176 & 32 & 75 & 23 & 191 & 0,041 & 419 \\
\hline CRR 2_BD & 581 & 2.877 .241 & 96 & 4.762 & 80 & 1,38 & 282 & 3,52 & 1.609 & 0,325 & 67 & 4.069 & 69 & 73 & 27 & 160 & 0,034 & 413 \\
\hline CRR 3_BB & 1.651 & 6.560 .002 & 97 & 4.001 & 70 & 1,57 & 373 & 5,32 & 1.476 & 0,337 & 64 & 4.381 & 59 & 78 & 22 & 151 & 0,039 & 532 \\
\hline CRR 4_BB & 1.240 & 6.700 .000 & & 5.411 & 80 & 1,77 & 330 & 4,13 & 1.720 & 0,317 & 69 & 3.957 & 61 & 84 & 14 & 122 & 0,023 & 281 \\
\hline CRR 5.1_BD & 1.257 & 9.136 .222 & 94 & 6.901 & 70 & 1,75 & 246 & 3,51 & 1.715 & 0,234 & 82 & 3.336 & 61 & 68 & 30 & 180 & 0,026 & 350 \\
\hline CRR 5.2_BD & 676 & 4.409 .975 & 90 & 5.796 & 65 & 1,80 & 286 & 4,40 & 1.489 & 0,231 & 77 & 3.551 & 53 & 69 & 30 & 133 & 0,023 & 317 \\
\hline CRR 6_BB & 114 & 594.752 & 98 & 4.976 & 75 & 1,37 & 277 & 3,71 & 2.045 & 0,400 & 51 & 5.336 & & 83 & 17 & 181 & 0,037 & 485 \\
\hline CRR 7_BD & 1.030 & 5.043 .889 & 98 & 4.792 & 102 & 2,11 & 435 & 4,28 & 1.777 & 0,364 & 76 & 3.587 & 27 & 70 & 30 & 159 & 0,033 & 320 \\
\hline CRR 8.1_BD & 935 & 4.676 .757 & 97 & 4.807 & 80 & 2,29 & 464 & 5,80 & 1.634 & 0,329 & 66 & 4.109 & 22 & 68 & 27 & 140 & 0,029 & 350 \\
\hline CRR 8.2_BD & 343 & 1.798 .395 & & 5.206 & 90 & 5,13 & 1.013 & 11,25 & 1.755 & 0,336 & 73 & 3.736 & 11 & 68 & 31 & 172 & 0,032 & 359 \\
\hline CRR 9.1_BD & 168 & 1.656 .076 & 85 & 8.483 & 77 & 5,44 & 550 & 7,56 & 2.851 & 0,273 & 73 & 3.736 & 42 & 64 & 35 & 272 & 0,028 & 361 \\
\hline CRR 9.2_BD & 121 & 548.514 & 80 & 4.064 & 71 & 5,60 & 1.046 & 14,82 & 1.666 & 0,297 & 67 & 4.208 & 12 & 49 & 51 & 272 & 0,053 & 652 \\
\hline CRR 9.3_BD & 134 & 600.604 & 93 & 4.242 & 75 & 5,20 & 1.156 & 15,49 & 1.361 & 0,288 & 72 & 3.856 & 9 & 42 & 57 & 226 & 0,048 & 645 \\
\hline CRR 9.4_BD & 752 & 2.294 .661 & 92 & 2.787 & 65 & 1,18 & 392 & 6,03 & 825 & 0,289 & 62 & 4.442 & 24 & 70 & 29 & 72 & 0,025 & 390 \\
\hline CRR 10.1_BD & 855 & 3.168 .612 & 92 & 3.397 & 82 & 2,08 & 571 & 6,97 & 1.126 & 0,305 & 73 & 3.722 & 26 & 71 & 29 & 109 & 0,032 & 361 \\
\hline CRR 10.2_BB & 572 & 1.759 .553 & 91 & 2.786 & 104 & 2,50 & 823 & 7,91 & 1.230 & 0,401 & 71 & 3.853 & 34 & 91 & 9 & 75 & 0,027 & 237 \\
\hline CRR 10.3_BB-BD & 1.020 & 2.281 .779 & 88 & 1.968 & 130 & 2,63 & 1.189 & 9,14 & 1.141 & 0,511 & 70 & 3.931 & 20 & 66 & 33 & 118 & 0,060 & 406 \\
\hline CRR 10.4_BB & 770 & 3.158 .242 & 81 & 3.278 & 106 & 2,62 & 673 & 6,35 & 1.475 & 0,364 & 79 & 3.437 & 41 & 82 & 16 & 110 & 0,034 & 257 \\
\hline CRR 11.1_BD & 632 & 1.476 .002 & 100 & 2.325 & 66 & 1,63 & 697 & 10,56 & 924 & 0,396 & 45 & 5.993 & 32 & 66 & 27 & 93 & 0,040 & 605 \\
\hline CRR 11.2_BD & 349 & 834.589 & 95 & 2.277 & 56 & 1,72 & 719 & 12,84 & 865 & 0,362 & 42 & 6.455 & 26 & 53 & 36 & 108 & 0,047 & 803 \\
\hline CRR 11.3_BD & 1.735 & 4.025 .124 & 98 & 2.271 & 75 & 1,01 & 437 & 5,83 & 907 & 0,391 & 52 & 5.212 & 43 & 75 & 22 & 73 & 0,032 & 421 \\
\hline CRR 11.4_BD & 647 & 1.086 .973 & 91 & 1.532 & 64 & 1,39 & 828 & 12,94 & 775 & 0,461 & 38 & 7.208 & 26 & 63 & 36 & 83 & 0,054 & 776 \\
\hline CRR 11.5_BD & 122 & 267.894 & 97 & 2.136 & 68 & 4,10 & 1.866 & 27,45 & 898 & 0,409 & 45 & 6.019 & 13 & 36 & 63 & 155 & 0,073 & 1.041 \\
\hline CRR 12.1_BD & 3.100 & 13.946 .669 & 99 & 4.384 & 60 & 0,73 & 173 & 2,88 & 862 & 0,195 & 84 & 3.253 & 62 & 88 & 12 & 72 & 0,016 & 268 \\
\hline CRR 12.2_BD & 2.962 & 13.316 .132 & 99 & 4.410 & 61 & 0,99 & 230 & 3,75 & 897 & 0,202 & 83 & 3.289 & 50 & 87 & 13 & 76 & 0,017 & 275 \\
\hline CRR 12.3_BD & 3.265 & 13.262 .484 & 98 & 3.949 & 55 & 0,77 & 198 & 3,61 & 737 & 0,183 & 82 & 3.330 & 52 & 87 & 13 & 62 & 0,015 & 276 \\
\hline Valor $n$ & náximo & & 100 & 8.483 & 130 & 5,60 & 1.866 & 27,45 & 2.851 & 0,511 & 84 & 7.208 & 69 & 91 & 63 & 272 & 0,073 & 1.041 \\
\hline Valor $p$ & promed & & 93 & 4.173 & 76 & 2,23 & 566 & 7,46 & 1.403 & 0,325 & 66 & 4.333 & 37 & 71 & 28 & 140 & 0,035 & 446 \\
\hline Valor $n$ & nínimo & & 80 & 1.532 & 55 & 0,73 & 173 & 2,88 & 737 & 0,183 & 38 & 3.253 & 9 & 36 & 9 & 62 & 0,015 & 237 \\
\hline Desviac & ción típ & pica & 6 & 1.603 & 17 & 1,47 & 402 & 5,45 & 497 & 0,078 & 12,9 & 1.029 & 18 & 13 & 13 & 58 & 0,013 & 191 \\
\hline Coefici & iente $\mathrm{d}$ & le variación & 0,21 & 0,38 & 0,22 & 0,66 & 0,71 & 0,73 & 0,35 & 0,24 & 0,19 & 0,24 & 0,48 & 0,19 & 0,47 & 0,41 & 0,38 & 0,43 \\
\hline
\end{tabular}

El análisis de las 12 CC.RR. representa una superficie promedio regada anual de 28.176 ha. El indicador hídrico más representativo es el consumo de agua por unidad de área regada (VsSr) que arroja un valor promedio de $4.173 \pm 1.603 \mathrm{~m}^{3} \cdot \mathrm{ha}^{-1}$, situándose por debajo del suministro bruto de $6.888 \mathrm{~m}^{3} \cdot \mathrm{ha}^{-1}$ para CyL, precedente al proceso de modernización (fuente: Plan Nacional de Regadíos Horizonte 2008).

Se comenta brevemente los indicadores energéticos más representativos: 
- Índice de carga (ICE): todas las CC.RR. bombean, ya sea a balsa o directamente a red, con presiones que van desde los 130 mca hasta los $55 \mathrm{mca}$. El valor promedio del ICE es $76 \pm 17$ mca. Resaltar que todos los bombeos se realizan desde captaciones superficiales (lo más deseable desde un punto de vista energético) en contraste con circunstancias de otras CC.RR. (zona de Levante y Castilla La Mancha) en el que el aporte extra de energía en forma presión es necesario por tener las captaciones de agua en el subsuelo. En todo caso, muchas de las regulaciones que se llevan a cabo se hace por presión constante, por lo que existe un alto margen de mejora en la mayoría de las CC.RR.

- Potencia contratada por unidad de área regada (NcSr) y por volumen de agua suministrado (NcVs): el valor promedio de $\mathrm{NcSr}$ es de $2,23 \pm 1,47 \mathrm{~kW} \cdot \mathrm{ha}^{-1}$ lo que representa una gran variación entre CC.RR., y algo mayor del nivel de referencia de $2 \mathrm{~kW} \cdot \mathrm{ha}{ }^{-1}$ que requiere una modernización, según recoge la bibliografía existente. Se constata que dentro de la misma C.RR., conforme entra en riego más superficie porque los regantes van amueblando sus parcelas, el valor de NcSr se reduce. En cuanto a NcVs el valor promedio es de $566 \pm 402 \mathrm{~kW} \cdot \mathrm{hm}^{-3}$ y su comportamiento es paralelo al de $\mathrm{NcSr}$.

- Índice de potencia contratada (INc): el valor promedio de INc es $7,46 \pm 5,45 \mathrm{~kW} \cdot \mathrm{hm}^{-3} \cdot \mathrm{ha}^{-1}$. Este índice es el que presenta mayor CV.

- Energía activa total consumida por unidad de área regada (EaSr) y por volumen de agua que entra en el sistema $\left(\mathrm{EaV}_{\mathrm{T}}\right)$ : el valor promedio de $\mathrm{EaSr}$ es de $1.403 \pm 497 \mathrm{kWh} \cdot \mathrm{ha}^{-1}$, cuantía menor con respecto a los $1.560-1.600 \mathrm{kWh} \cdot \mathrm{ha}^{-1}$ que figura en la bibliografía como valor medio del regadío español. Aún así, todas las CC.RR. se encuadran como grandes consumidoras ( $\mathrm{EaSr}>1.000 \mathrm{kWh} \cdot \mathrm{ha}^{-1}$ ) según la clasificación propuesta por el IDAE. Respecto a $\mathrm{EaV}_{\mathrm{T}}$ el valor promedio es de $0,325 \pm 0,078 \mathrm{kWh} \cdot \mathrm{m}^{-3}$, situándose dentro del rango de los $0,28-0,34 \mathrm{kWh} \cdot \mathrm{m}^{-3}$ que figura en la bibliografía como estimación del riego en España.

- Eficiencia energética de bombeo (EEB): el valor promedio de EEB es del $66 \pm 13 \%$ por lo que la clasificación energética según el protocolo del IDAE se corresponde con el tipo $A$ (excelente para EEB > 65\%).

- Índice de carga de energía activa consumida (IEa): el valor promedio de IEa es de $4.333 \pm 1.029 \mathrm{kWh} \cdot \mathrm{hm}^{-3} \cdot \mathrm{mca}^{-1}$. Existe una relación directa entre IEa y EBB. Los valores mayores de EEB implican los menores valores de IEa.

En cuanto a los indicadores más representativos que tienen en cuenta los costes de energía y de potencia contratada (sumatorio de todas las facturas del año incluyendo el impuesto eléctrico y sin contar el IVA) destacamos los siguientes:

- Gasto energético por energía (GCea) y por potencia (GCep): el valor promedio de GCea es del $71 \pm 13 \%$ y el de GCep es del $28 \pm 13 \%$, lo que nos da una idea del porcentaje de la factura eléctrica total que se destina al pago de energía consumida y a la potencia contratada.

- Coste energético por área regada (CENSr) y por volumen suministrado (CENVs): el valor promedio de CENSr es de $140 \pm 58 € \cdot$ ha $^{-1}$ y el de GCep es de $0,035 \pm 0,013 € \cdot \mathrm{m}^{-3}$, valores considerados no excesivos con respecto a regadíos de otras geografías españolas.

- Índice de coste energético (ICEN): el valor promedio de ICEN es de $446 \pm 191 € \cdot \mathrm{hm}^{-3} \cdot \mathrm{m}^{-3}$. El menor valor de este índice de obtiene en la CRR 10.2_BB pese a que tiene un índice de carga energética (ICE) bastante alto, de 104 mca. Esta C.RR obtiene el mayor valor de GCea (91\%), lo que podría indicar que la potencia contratada está muy bien escogida.

Por falta de espacio no se presenta la comparación de los resultados con los publicados en otros estudios de caracterización de CC.RR. de otras geografías españolas, y en todo caso, animamos a los investigadores a seguir realizando trabajos de esta índole y puestas en común de los resultados para poder realizar comparativas entre distintas zonas regables de España.

Del estudio de los indicadores se deduce que las 12 C.RR. analizadas pueden mejorar en los siguientes aspectos: 
- Aumento de la superficie regada anual con respecto a la superficie total regable disponible. El proceso de amueblamiento en parcela según qué zonas, es lento; o no todos los regantes hacen uso de las nuevas infraestructuras, dándose casos en los que conviven los dos sistemas (riego por superficie y riego a presión). A mayor superficie regada anual muchos índices mejoran en valor absoluto.

- La presión de bombeo si bien está ajustada a las necesidades del sistema, cambiar su regulación (de punto fijo a curva de consigna por escalones, o incluso a dinámica) y/o reconfigurar la secuencia de activación/parada de los grupos mejoraría los valores obtenidos de los índices.

- La potencia contratada en la mayoría de las situaciones se puede ajustar mejor, si bien incurriría en cambios en la organización de los riegos (concentración, riego nocturno, aprovechamiento de todas las horas disponibles P6...).

Finalmente resaltar que la CRR 12 se identifica con la CRRCPB, evaluada como zona piloto en el proyecto OPTIREG y en la que el Grupo Tragsa realiza trabajos de gestión y mantenimiento. Esta C.RR. es de las que obtiene los mejores resultados en la mayoría de los índices evaluados.

\section{6- Estudios energéticos en zonas piloto}

Otro pilar de trabajo del grupo WP3 es estudiar medidas de eficiencia energética enfocadas en la gestión de los riegos que se realiza durante la campaña de riegos. Para ello se cuenta con dos CC.RR. emplazadas en la provincia de León y en la que el grupo Tragsa realiza la gestión, el mantenimiento e incluso la compra de energía en mercado del pool eléctrico: la CRRMIP de 12.499 ha de superficie regable y 5.700 ha de superficie regada anual, dividida en varios sectores; y la CRRCPB que cuenta con 15.957 ha de superficie regable y 13.090 ha de superficie anual regada, también fragmentada en varios sectores.

Los trabajos desarrollados han sido la elaboración de un modelo hidráulico completo de un sector de riego en cada una de las dos zonas piloto con el programa GESTAR: sector XI en la CRRMIP que abarca 1.592 ha, cuenta con 153 hidrantes, $43.353 \mathrm{ml}$ de tubería (PVC-U y PRFV) y una EB de inyección directa a red compuesta por un total de 9 bombas de diversos tamaños con presiones de bombeo desde los 48 mca hasta los $55 \mathrm{mca}$; y el sector I de la CRRPB que abarca 3.951 ha, cuenta con 318 hidrantes, $86.287 \mathrm{ml}$ de tubería (PVC-U y PRFV) y una EB de inyección directa a red compuesta por un total de 12 bombas de diversos tamaños con presiones de bombeo desde los $48 \mathrm{mca}$ hasta los $55 \mathrm{mca}$.

En ambos sectores la presión a servir en los hidrantes que riegan es de 50 mca. Cuentan con un sistema de telecontrol que comanda la apertura y cierre de las electroválvulas y realiza el contaje del caudal promedio de cada riego mediante un emisor de pulsos insertado en el contador. El funcionamiento en cuanto a la organización de los riegos en ambos sectores es similar: el caso general es que cada regante solicite el riego por teléfono con una antelación de 48 horas en el que indica la hora del comienzo y la duración del riego de sus parcelas. El gestor con ayuda del PROGAR (programa de gestión de riegos) organiza las peticiones buscando la máxima eficiencia: no saturación de las líneas de transporte, concentración de caudales para los puntos de funcionamiento de las bombas con mejor rendimiento y no sobrepasar la potencia contratada en los distintos tramos horarios. Además, hay particulares como el riego por gravedad en el caso de la red del sector XI de la CRRMIP a ciertos hidrantes, o turnos fijos en algunos hidrantes que se han establecido con aprobación del regante.

En cualquier caso, se dispone de todos los registros de cada escenario de riego que se vaya produciendo a lo largo de la campaña de riegos, históricos necesarios para calibrar y evaluar el modelo hidráulico de la red con fines a proponer mejoras mediante escenarios simulados: tanto en la red (qué hidrantes han abierto y a qué caudal promedio han regado, 
así como registro de presiones en varios hidrantes en donde se hayan instalado transductores y sondas); y en la EB (presión reinante en los colectores de aspiración e impulsión, caudales bombeados y kWh consumidos).

El modelo de cada red debe de reproducir mediante un programa de simulación (en este caso GESTAR) el comportamiento real del sistema físico que representa con la mayor exactitud posible. Se incide en este punto en la importancia que, para la bondad del modelo, tiene la obtención de una información precisa de los elementos que componen el sistema de distribución a modelar. La bondad del modelo depende muy directamente de la precisión de los datos de partida. El proceso de elaborar cada modelo ha supuesto recopilar información de todos los elementos significativos y existentes de la red y tratarla para poder ser asimilada por el programa de análisis y simulación, provenientes principalmente del proyecto de liquidación de cada obra (As-built) y de datos actualizados facilitados por el gestor:

- Topología de la red. Toda esta información se obtiene de los planos reales de la red y del GIS para posicionar las coordenadas X E Y de todos los nodos.

- Conducciones. Hay que conocer diámetro interior, longitud, conectividad entre nodos y material. Se establece como rugosidad absoluta los siguientes valores: $0,007 \mathrm{~mm}$ para el PVC-U, 0,015 mm para los tubos de PRFV y 0,1 $\mathrm{mm}$ para los tubos de acero.

- Bombas. Se debe conocer para cada uno de los equipos de bombeo su potencia, curvas características obtenidas de los ensayos de fábrica, conectividad y niveles de aspiración.

- Puntos de consumo. Cota reales de los hidrantes según los planos de perfiles longitudinales de los ramales (se trata de la cota del terreno a la que se le suma en todos los casos $35 \mathrm{~cm}$ ), dotación asignada, conectividad a la red y presión de consigna.

Se ha realizado una esqueletización de la red que ha consistido en una simplificación del entramado de tuberías entre la principal y el hidrante (los hidrantes vienen pinchados sobre la propia tubería principal), y no se ha considerado ventosas, válvulas de seccionamiento, válvulas antiretorno, filtros, reducciones, ampliaciones y caudalímetros para los elementos instalados en la EB. Además, se crean dos modelos diferentes, en que se distingue en uno de ellos la EB completa (están modelizadas todas las bombas instaladas junto con sus colectores individuales de aspiración e impulsión); y otro modelo en el que se sustituye el conjunto de bombas y colectores individuales por una pseudo-bomba que engloba el comportamiento de todas y que viene caracterizada por la nube de puntos $H-Q$, $P-Q$ y Rto-Q, extraídos del SCADA (véase más adelante).

Figura 1. Plano constructivo de planta de la red del sector I de la CRRCPB (izquierda) y modelo hidráulico creado de la misma red (derecha) mediante el programa GESTAR
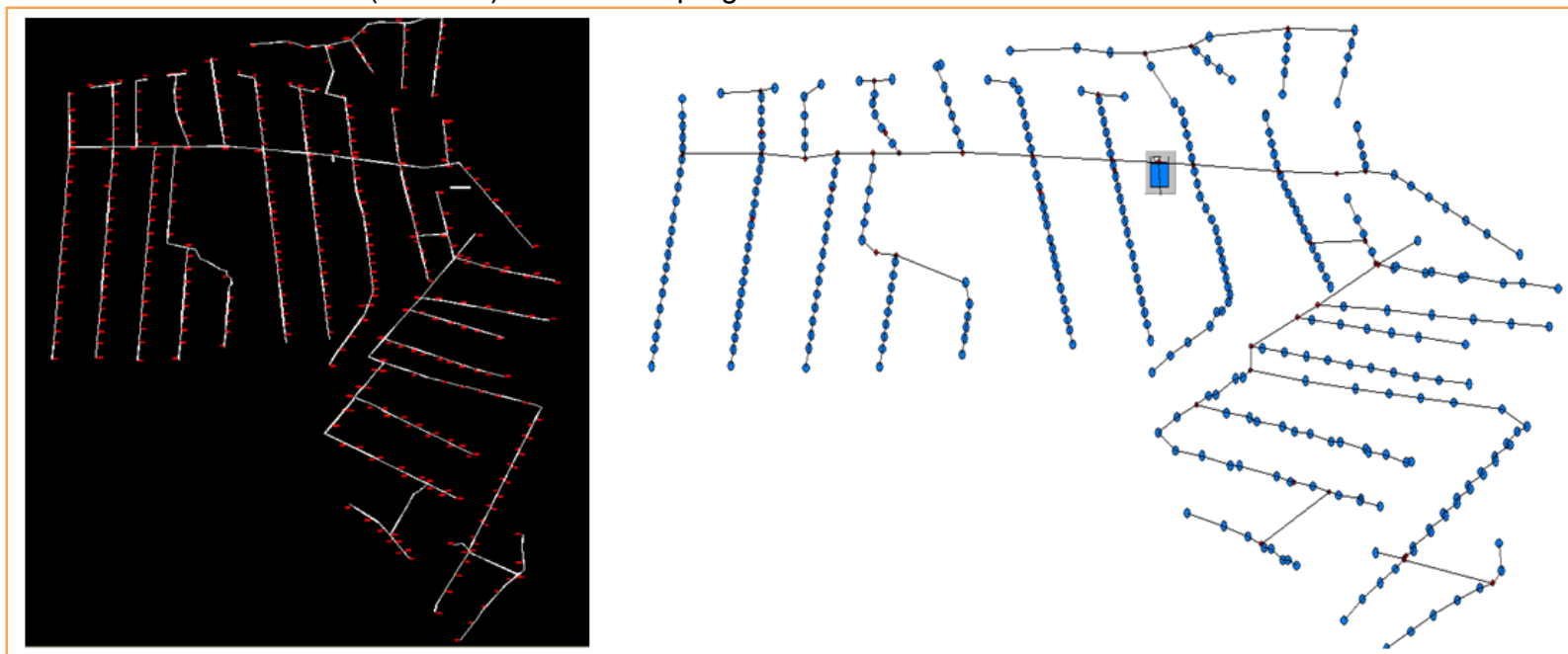

Se efectúa una primera calibración en estática de cada modelo de red, en el que se compara los valores de presión obtenidos de los transductores que hay instalados en varios hidrantes 
(el valor de la presión se considera como el promedio de los datos transmitidos por el telecontrol cada media hora), con la calculada con el modelo, en dos días que no hubo riego y la red estaba parada. Además de los datos de la presión suministradas en los hidrantes de la red por los transductores es necesario conocer la presión al inicio de la misma, tomada del colector de impulsión.

De los resultados encontramos que las diferencias de presión obtenidas entre el modelo y los transductores instalados en campo no son mayor a $2 \mathrm{mca}$, por lo que la precisión de las cotas es válida. A continuación se llevó a cabo una calibración en dinámica con apoyo de los históricos que almacena PROGAR y el SCADA de la EB en el que se analizan varios escenarios de bombeo (caudales desde los $100 \mathrm{l} / \mathrm{s}$ hasta los $4.000 \mathrm{l} / \mathrm{s}$ ) y procediendo de la misma manera (comparar los valores de los transductores de presión con las presiones calculadas con el modelo para cada escenario de riego) se llega a resultados muy positivos, en el sentido de que las diferencias encontradas son muy estrechas, con un valor promedio entero de las diferencias entre campo y modelo de 0,82 mca. En conclusión, hemos obtenido un modelo de red con una buena calibración de partida, por lo que los resultados obtenidos en las simulaciones que se realicen a efectos de analizar estrategias de gestión (sin tener que llegar a experimentarlos físicamente) serán fácilmente contrastados y validados en la realidad. Además, se ha calculado con la metodología propuesta por el equipo GESTAR la curva de consigna recomendada (en adelante CCR) de cada una de las redes modelizadas, al objeto de poder compararla con la curva motriz de cada EB y analizar la calidad del servicio y el rango de mejora.

Tan importante es obtener una red bien calibrada, como modelizar el comportamiento real de la EB que suministra el caudal y la presión a ésta. Para conseguir este objetivo, se ha realizado un análisis de los datos proporcionados por el SCADA de la EB durante varios días de riegos de la campaña 2014 y que presentara un gran abanico de valores. En el caso del sector I de la CRRCPB, la presión de bombeo es variable en función del caudal, y ésta viene configurada mediante unos escalones de bombeo programados en el SCADA que gobierna la estación, por lo que se obtiene una nube de puntos $H-Q, P-Q$ y Rto-Q. La metodología empleada ha sido obtener los registros continuos (1 valor cada segundo) de la presión en la aspiración, la presión en la impulsión y el caudal bombeado, y realizar promedios horarios, ya que los valores suministrados por el analizador de redes del consumo energético (kWh) es horario. Conviene indicar que el analizador de redes se encuentra en el cuadro general de la instalación, por lo que la energía registrada incluye todas las pérdidas de la instalación (cableado eléctrico, rendimientos de los motores, bombas, arrancadores y variadores) y los consumos de los servicios auxiliares (sondas, ordenador, ventilación forzada, iluminación, filtro general, automatismos, caudalímetros, motores de las válvulas...).

La figura 2 muestra la nube de puntos $\mathrm{H}-\mathrm{Q}$ de la EB del sector I de la CRRCPB y la $\mathrm{CCR}$ de la red, cuya expresión cuadrática viene dada por $\mathrm{H}(\mathrm{mca})=52,14+0,178 \cdot \mathrm{Q}^{2}\left(\mathrm{~m}^{3} / \mathrm{s}\right)$. 
Figura 2. Nube de puntos $H-Q$ de la EB y CCR de la red del sector I de la CRRCPM

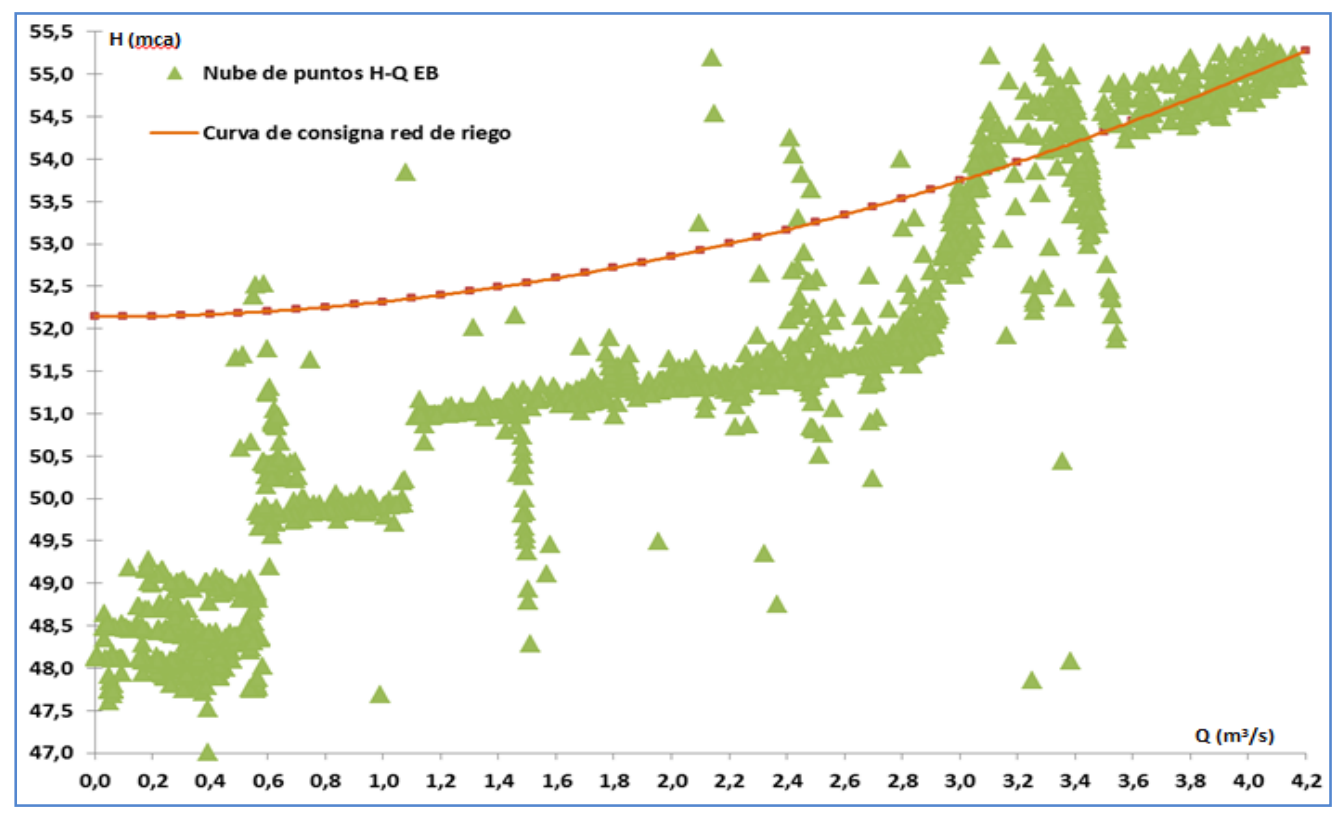

De la figura 2 observamos, que efectivamente, la EB funciona con escalones de bombeo, por lo ya que supone un ahorro energético en vez de bombear a punto fijo, como se hace en la muchas de la CC.RR. analizadas. La CCR está a la par con la curva motriz a partir de los $3.600 \mathrm{l} / \mathrm{s}$ por lo que se está bombeando correctamente. Entre los 3.000 y 3.400 I/s se aprecia que se está bombeando por encima de la CCR, por lo que si disminuyera la presión de bombeo no conllevaría merma en la calidad del servicio, además de que se produciría un potencial ahorro energético. Entre los 3.400 y $3.600 \mathrm{l} / \mathrm{s}$ se observa que se produce un mal recubrimiento entre bombas, como también ocurre a los $1.500 \mathrm{l} / \mathrm{s}$ y a los $2.500 \mathrm{l} / \mathrm{s}$. Desde los $0 \mathrm{l} / \mathrm{s}$ hasta los $600 \mathrm{l} / \mathrm{s}$ se bombea por debajo de la CCR (3,5 mca menos) y entre los 600 hasta los $3.000 \mathrm{l} / \mathrm{s}$ se bombea con un promedio de 1,5 mca por debajo de la CCR. En conclusión, hasta los $3.000 \mathrm{l} / \mathrm{s}$ se bombea por debajo de la CCR, si bien con presiones no muy distantes a los valores de la curva, por lo que la calidad del servicio no se ve mermada. Además, es preciso ajustar la entrada/salida y estados de transición de bombas en ciertos caudales.

Además de los datos obtenidos de la nube de puntos $\mathrm{H}-\mathrm{Q}$, se ha confeccionado la nube de puntos $P-Q$ y Rto-Q y parametrizando todos los pares de datos, se obtiene las curvas que utilizaremos para modelizar la EB en GESTAR (pseudo-bomba). Con todo, obtenemos un modelo completo de red + EB del que se realizan los siguientes estudios y análisis mediante una plataforma de enlace entre PROGAR y TELEGESTAR de manera que las peticiones de riego realizadas y que configura una programación de riegos en PROGAR se carguen en GESTAR de forma automática:

- Simulación predictiva del estado de la red para programaciones de riego temporales. prediciendo todas las variables hidráulicas y energéticas que van a tener lugar en todos los puntos, al ejecutar una determinada programación de riegos.

- Determinar los consumos energéticos y el coste económico total de la programación, de manera que puedan acotarse y ensayar alternativas.

- Reorganización de las peticiones de riego mediante un algoritmo de optimización de tipo evolutivo.

- Supervisar el estado de la red en condiciones de operación con apoyo del telecontrol.

- Recalibración automática del modelo computacional de la red. 


\section{7- Estudios energéticos en CC.RR. colaboradoras}

A continuación se presentan resultados preliminares obtenidos en una de las C.RR. que se han prestado a colaborar en el proyecto de investigación. La zona, regable se denomina "Comunidad de Regantes Virgen del Aviso", en la provincia de Zamora.

Las características generales de la zona regable son:

- Superficie regable: 1.874 ha

- $\mathrm{N}^{\circ}$ de parcelas: 1.047

- $\mathrm{N}^{\circ}$ de regantes: 801

- Sistema de riego: Aspersión

- Cultivos principales de la zona: maíz, alfalfa, remolacha y cereal

Existe una toma en el río Duero que conduce el agua hasta una cántara sobre la que se ubica la EB y desde la que se impulsa el agua directamente a la red de riego. La presión mínima de suministro a cada una de las parcelas es como mínimo de 35 m.c.a. La EB dispone de 6 bombas de eje vertical de las cuales 5 unidades son de $725 \mathrm{~kW}$ ( 3 unidades con variador de velocidad) con un caudal nominal de $2.160 \mathrm{~m}^{3} / \mathrm{h}$ y una altura nominal de 90 m.c.a. y 1 bomba es de $315 \mathrm{~kW}$ (con variador de velocidad) con un caudal nominal de 1.080 $\mathrm{m}^{3} / \mathrm{h}$ y una altura nominal de 76 m.c.a. Se dispone de un transductor de presión en el colector de impulsión y el bombeo se realiza a una presión de consigna fija:

- Presión de consigna máxima: 7,5 bar (76,5 m.c.a.)

- Presión de consigna mínima: 7,4 bar (75,48 m.c.a.)

- Variación de presión consigna: 0,1 bar (1,02 m.c.a.)

A continuación se exponen los resultados obtenidos del estudio de los registros de caudal y presión de la EB para toda la campaña 2014.

\section{Estudio de caudales}

A partir de los registros de caudal proporcionados por el caudalímetro de ultrasonidos de la EB y almacenados en el histórico de datos del SCADA se han representado todos los caudales bombeados en la campaña de riego habiéndose registrado un total de 10.646 registros de caudal y un caudal máximo de 1.816.6 l/s el 25/07/2014 a las 23:04:29.

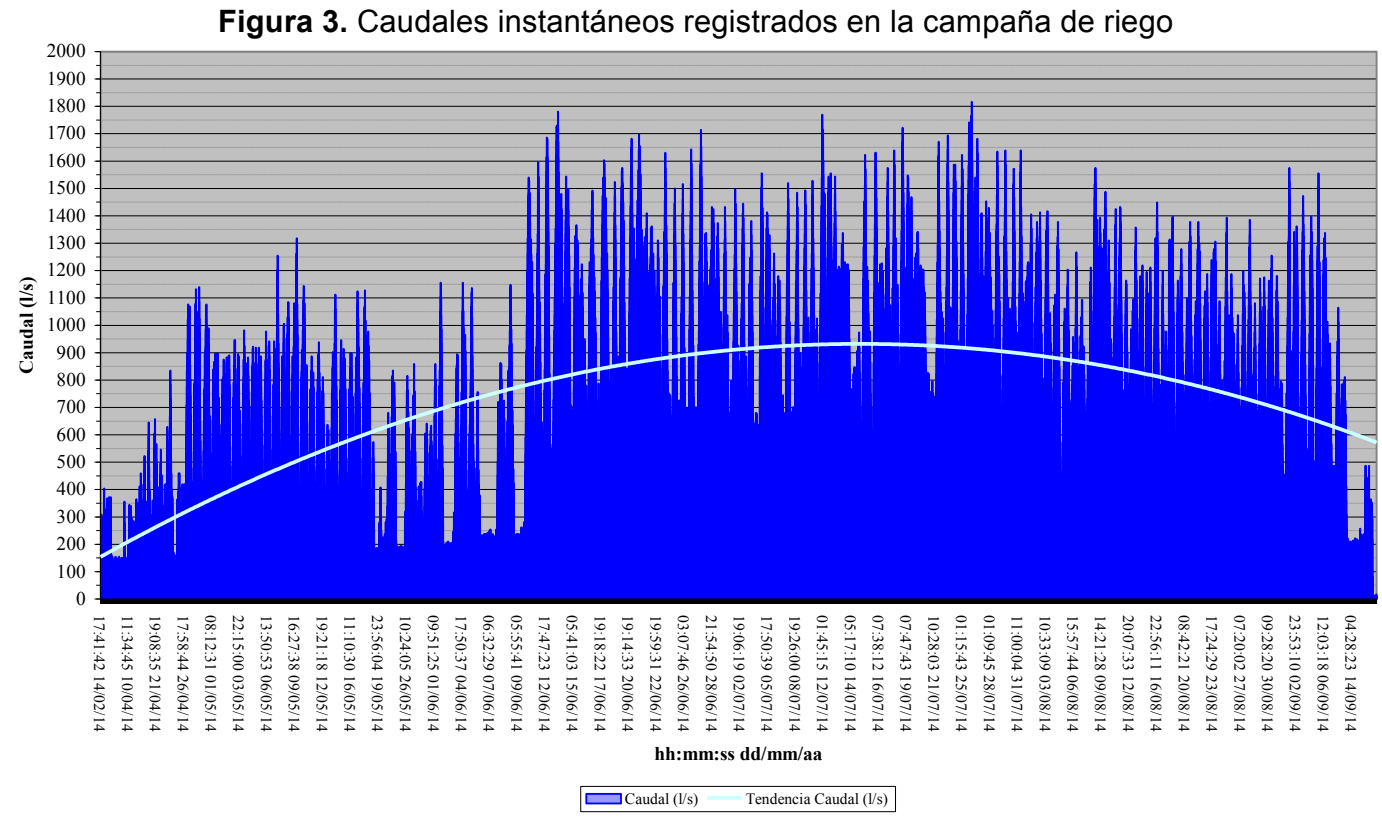

A partir de los caudales instantáneos registrados se han calculado unos caudales promedio horarios, dado que el número de registros por hora es más o menos constante, existiendo entre 6 y 10 datos por hora en los momentos de máximo caudal. En la figura 4 se 
muestra el caudal medio horario para cada una de las horas de la campaña de riego así como el caudal máximo y el mínimo horario.

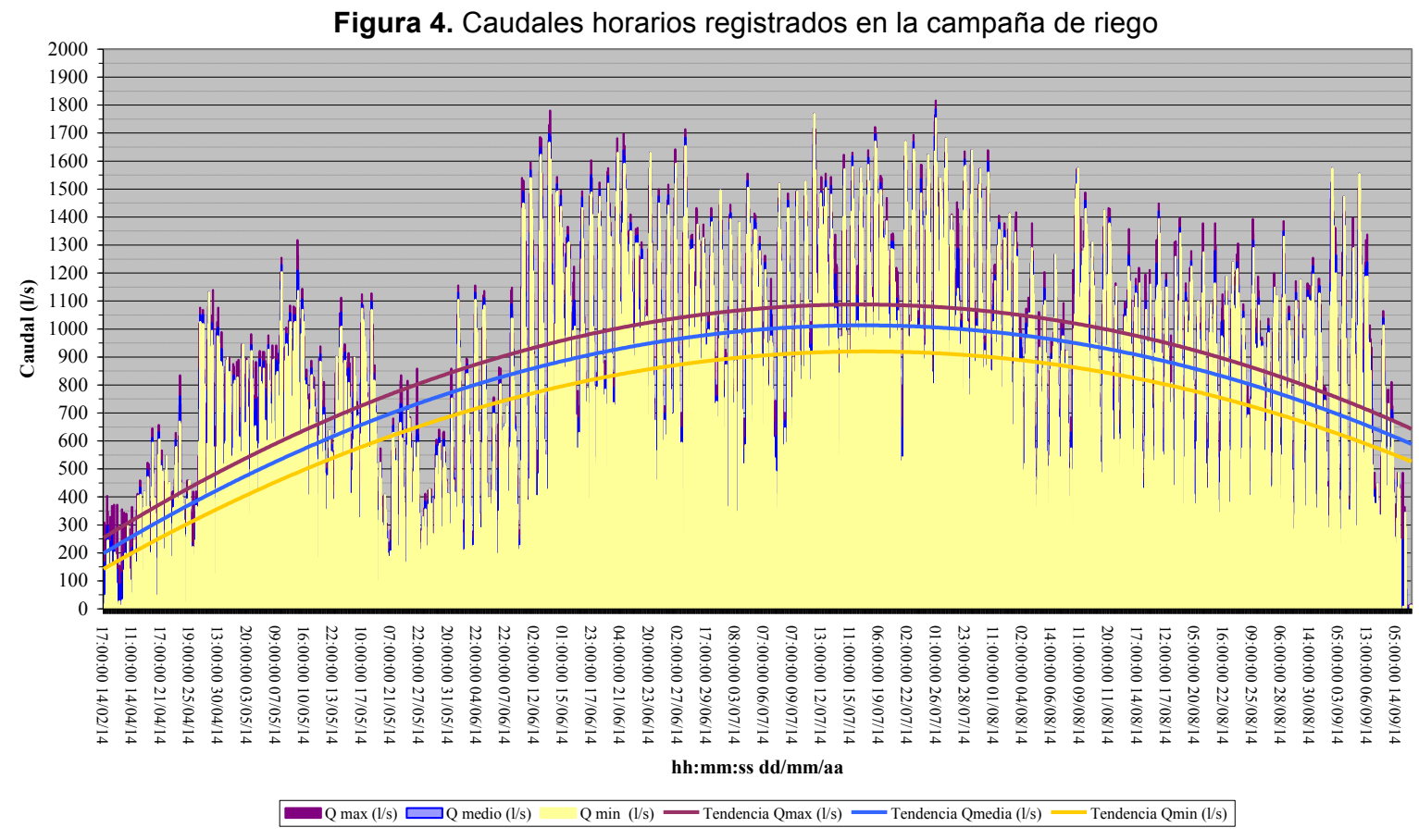

Se observa como existe poca diferencia entre el caudal máximo, el mínimo y el medio para una misma hora de bombeo

A partir de los caudales medios horarios se ha procedido al cálculo de la frecuencia de caudales en la campaña de riego. Se ha optado por emplear valores promedio horarios de caudal para evitar la distorsión en los resultados de frecuencia que podría provocar el tener un número distinto de registros de caudal en cada una de las horas de bombeo, de esta manera tenemos un solo registro para cada una de las horas de bombeo.

Se han establecido intervalos de caudal de $50 \mathrm{l} / \mathrm{s}$, desde los $0 \mathrm{l} / \mathrm{s}$ hasta los 1.850 I/s. Computando el número de registros cuyo valor de caudal se encuentran entre los intervalos de caudal definidos $\left(Q_{i}\right.$ y $\left.Q_{i+1}\right)$, se obtienen las frecuencias relativas de ocurrencia de los caudales. Estos registros se transforman en tiempo que el sistema ha estado trabajando en los intervalos de caudal considerados. 
Figura 5. Frecuencia relativa de ocurrencia de caudales con valores promedios horarios

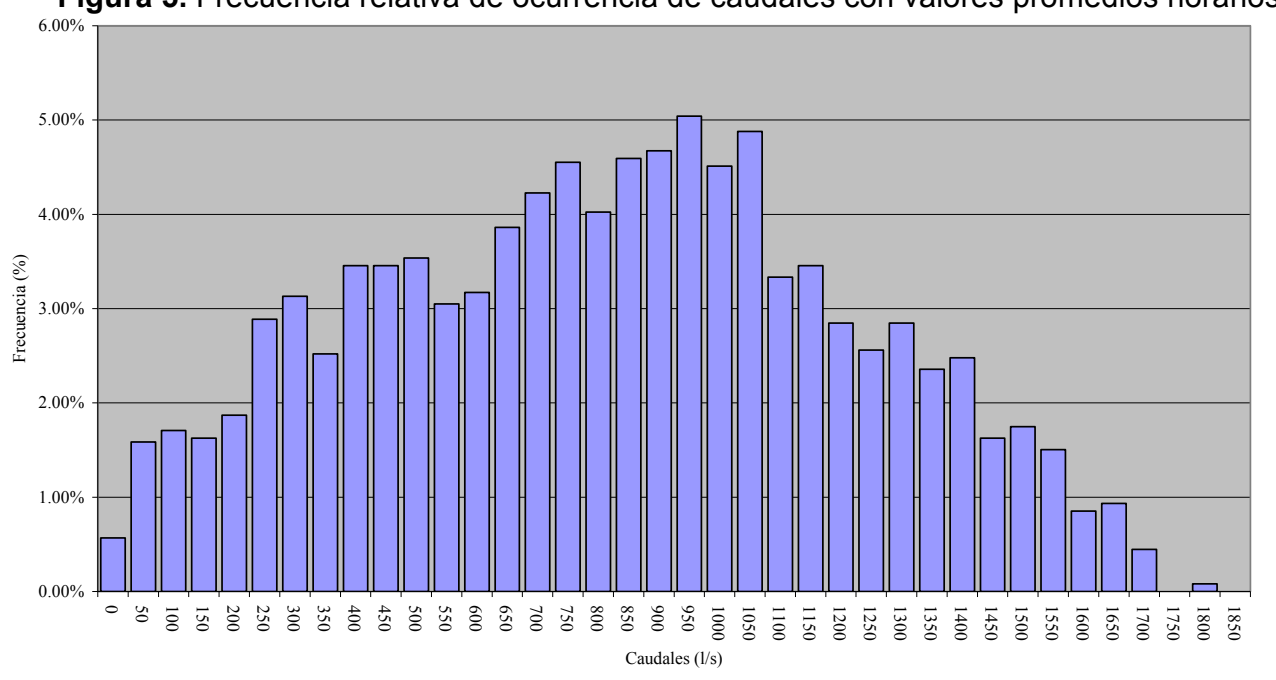

DFrecuencia caudales

A partir de la frecuencia de caudales podemos construir la Función de Densidad de Probabilidad (FDP). Así la probabilidad de que el caudal demandado $Q\left(_{i}\right)$ se encuentre en el rango $\left(Q_{i}, Q_{i+1}\right)$, será el área debajo de la curva. La FDP para un caudal q se define como la esperanza del tiempo relativo de existencia de un caudal comprendido entre q y q $+\mathrm{dq}$ :

$$
F D P(q)=\left[\frac{1}{T} \cdot \frac{d t}{d q}\right]=\frac{1}{T} \frac{[d t(q)]}{d q}
$$

siendo $T$, en segundos, la duración total de periodo de observación de caudales, y dt el tiempo de presencia de caudales comprendidos entre q y $q+\mathrm{dq}$.

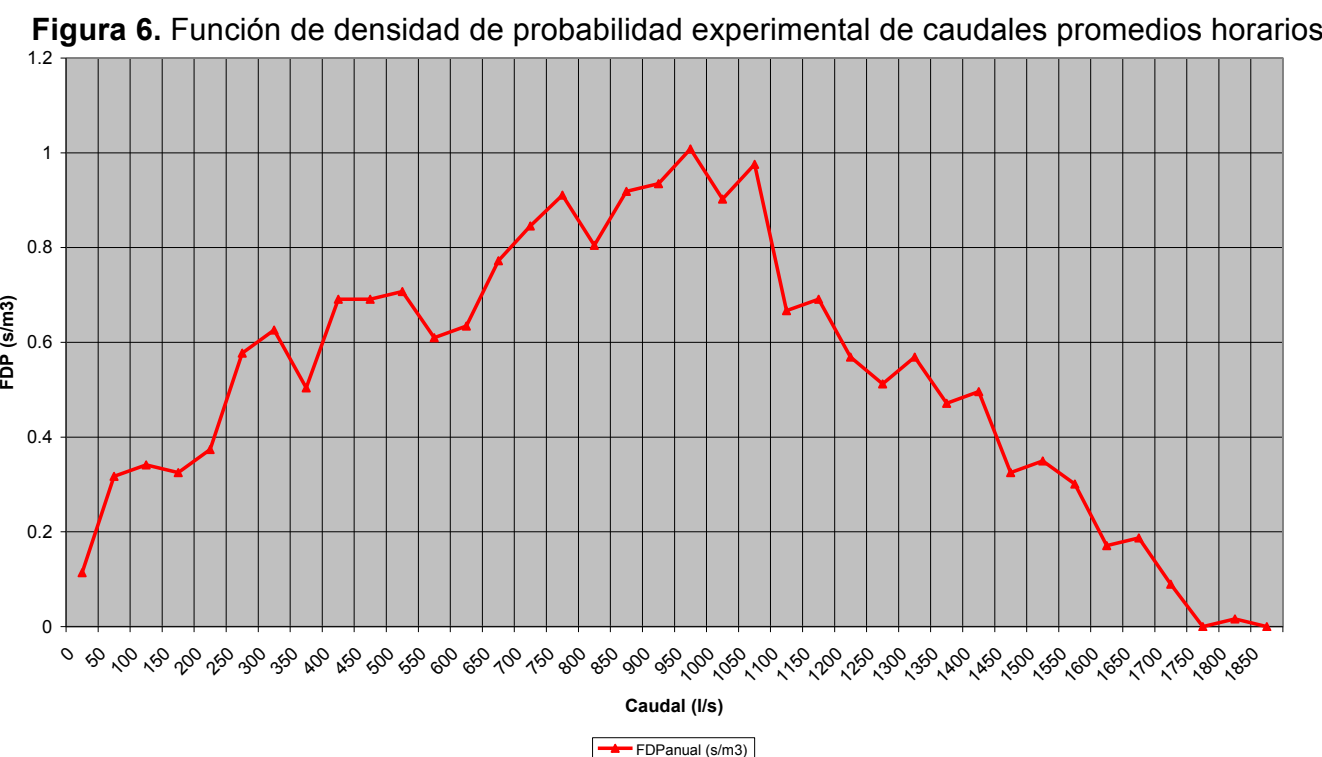

Por definición, toda FDP está normalizada y satisface:

$$
\int_{0}^{Q \max } F D P(q) \cdot d q=1
$$

Las curvas de la función de densidad de probabilidad mensual, reflejan el comportamiento de la distribución del consumo en cada mes (j) proporcionando la noción de la frecuencia de determinados caudales en la cabecera del sistema. 


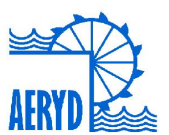

Figura 7. Curvas mensuales y anual de FDP de caudales promedios horarios

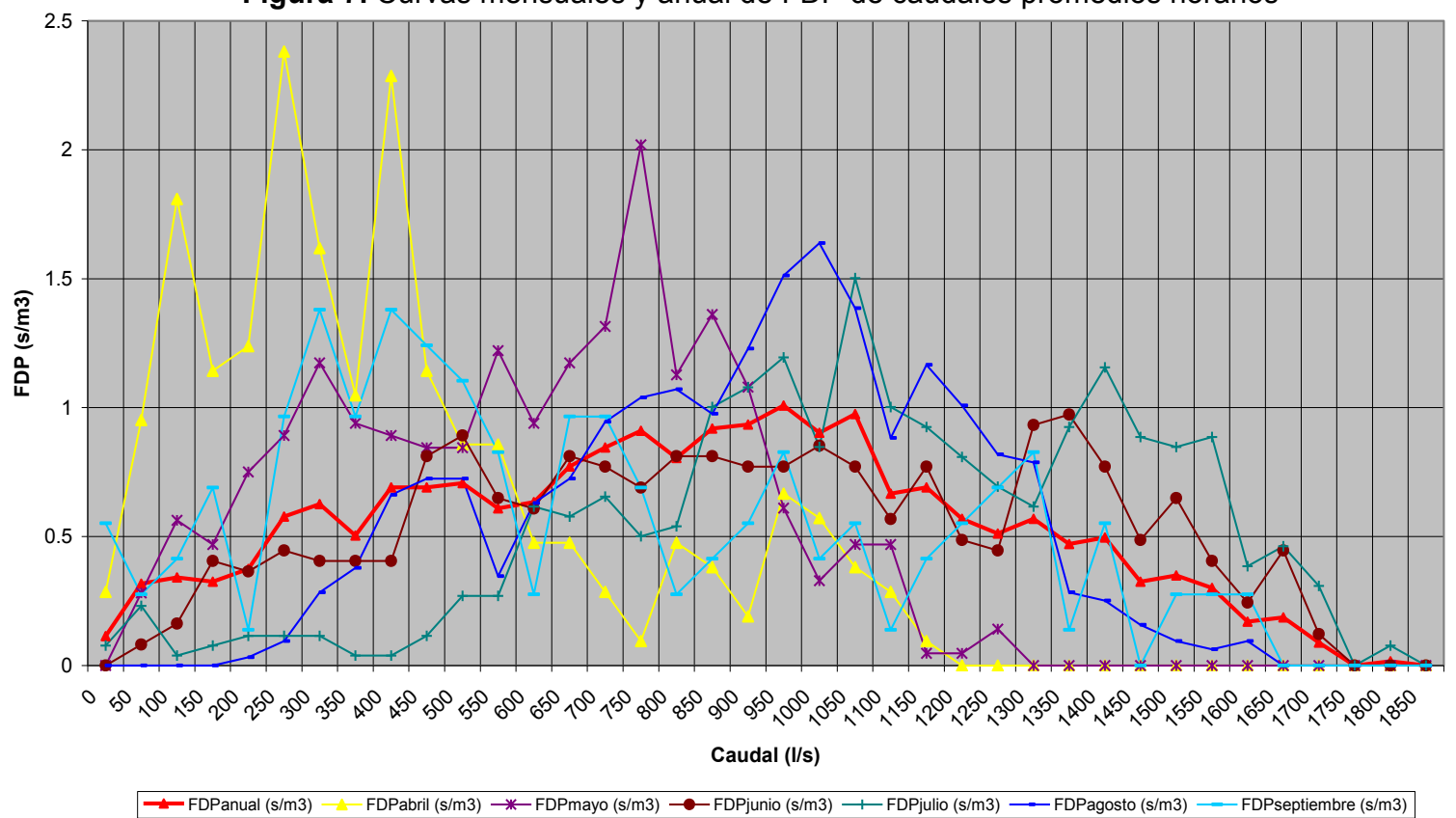

Nube de puntos de caudal y presión

A partir de los registros de caudal del caudalímetro, de los registros de presión del transductor ubicado en el colector de impulsión y de la sonda de nivel colocada en la cántara de aspiración, obtenemos una representación completa del funcionamiento de la EB a lo largo de la campaña de riego. A continuación se presentan las presiones horarias en el colector de impulsión a lo largo de toda la campaña de riegos.

Figura 8. Presiones horarias colector impulsión

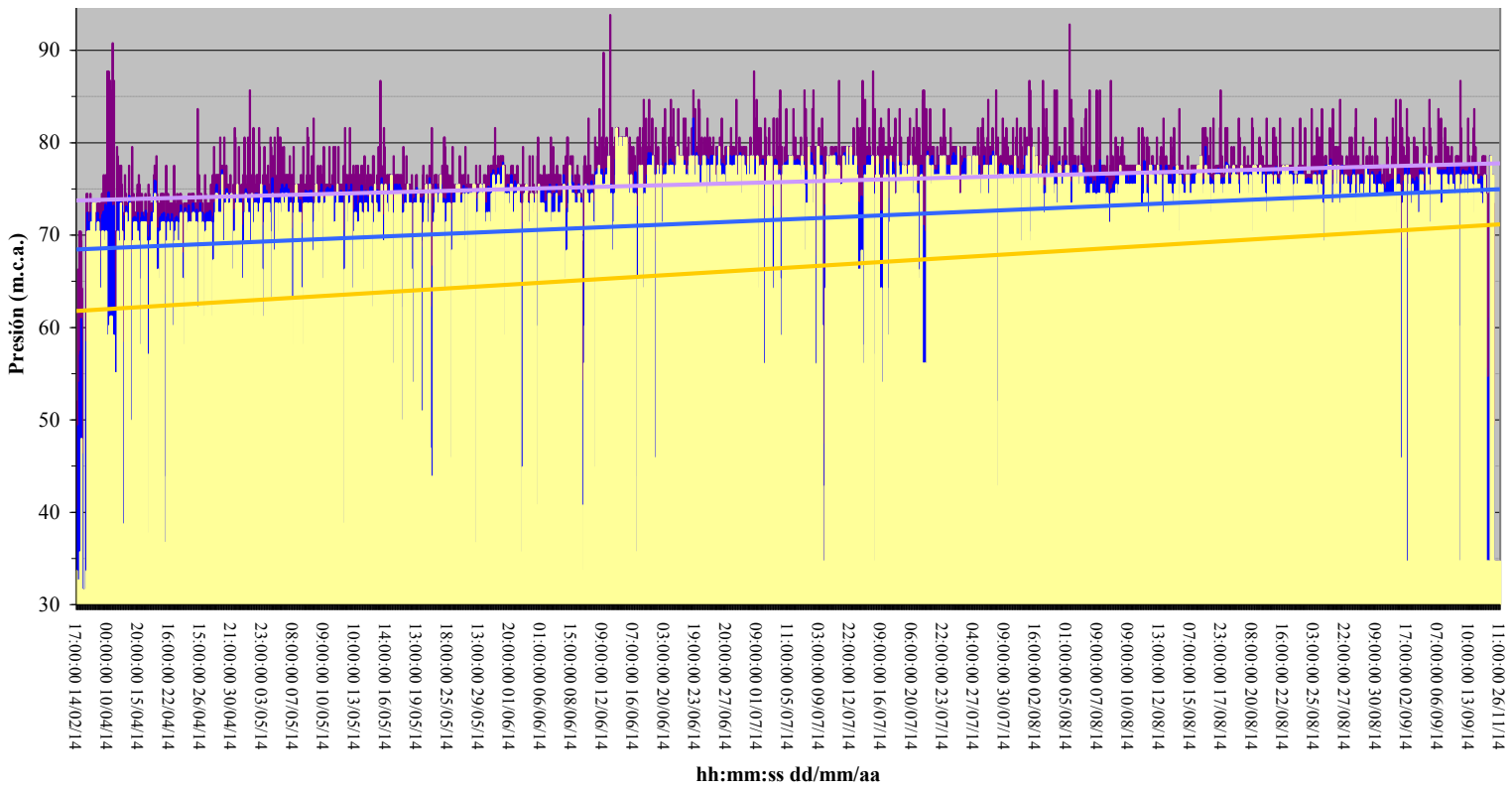

hh:mm:ss dd/mm/aa 


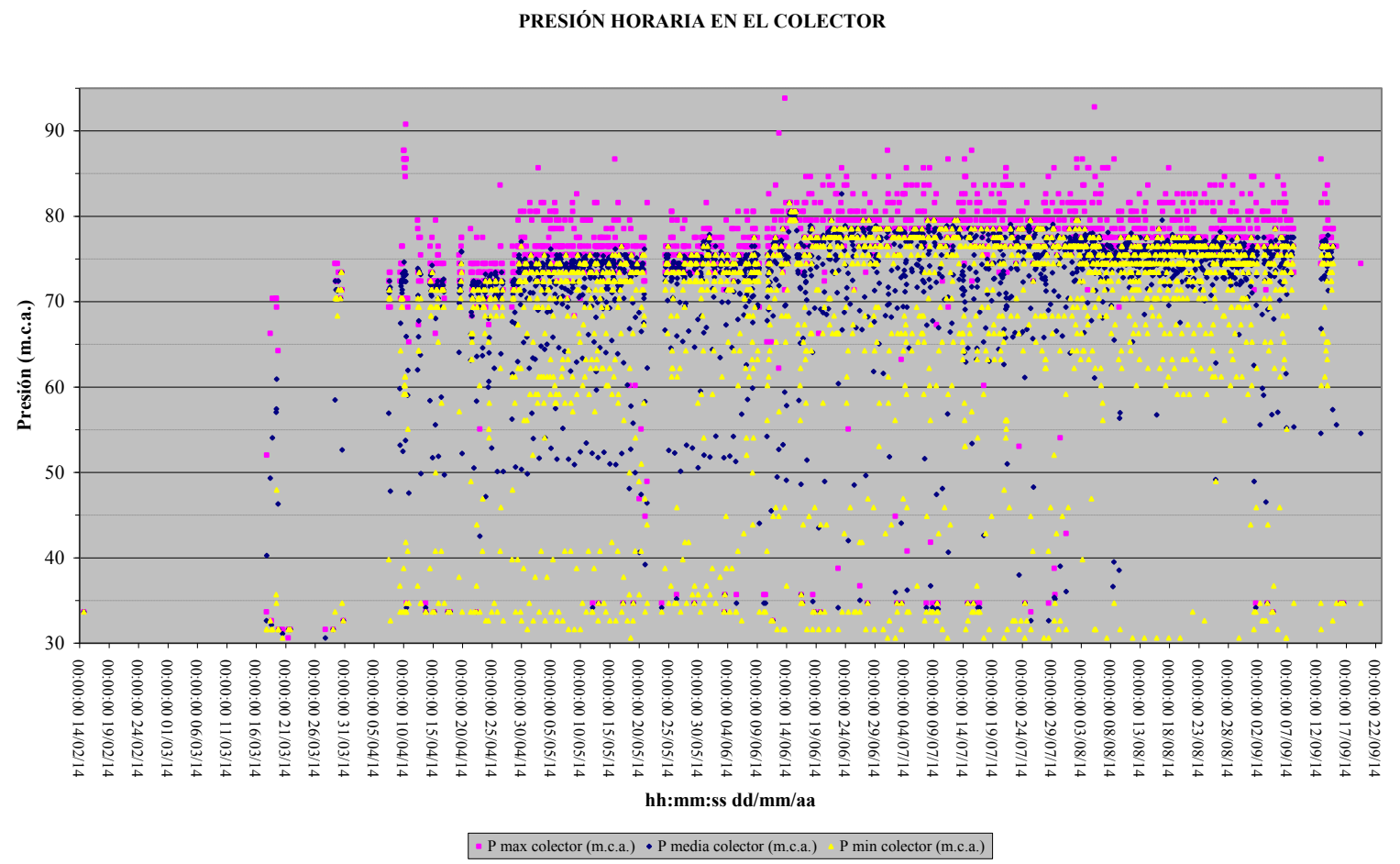

La representación del caudal bombeado, la presión registrada en el colector de impulsión y el nivel de agua en la cántara se muestra en la figura 9:

Figura 9. Registro completo de variables en la EB: presión impulsión, caudal bombeado y nivel de cántara

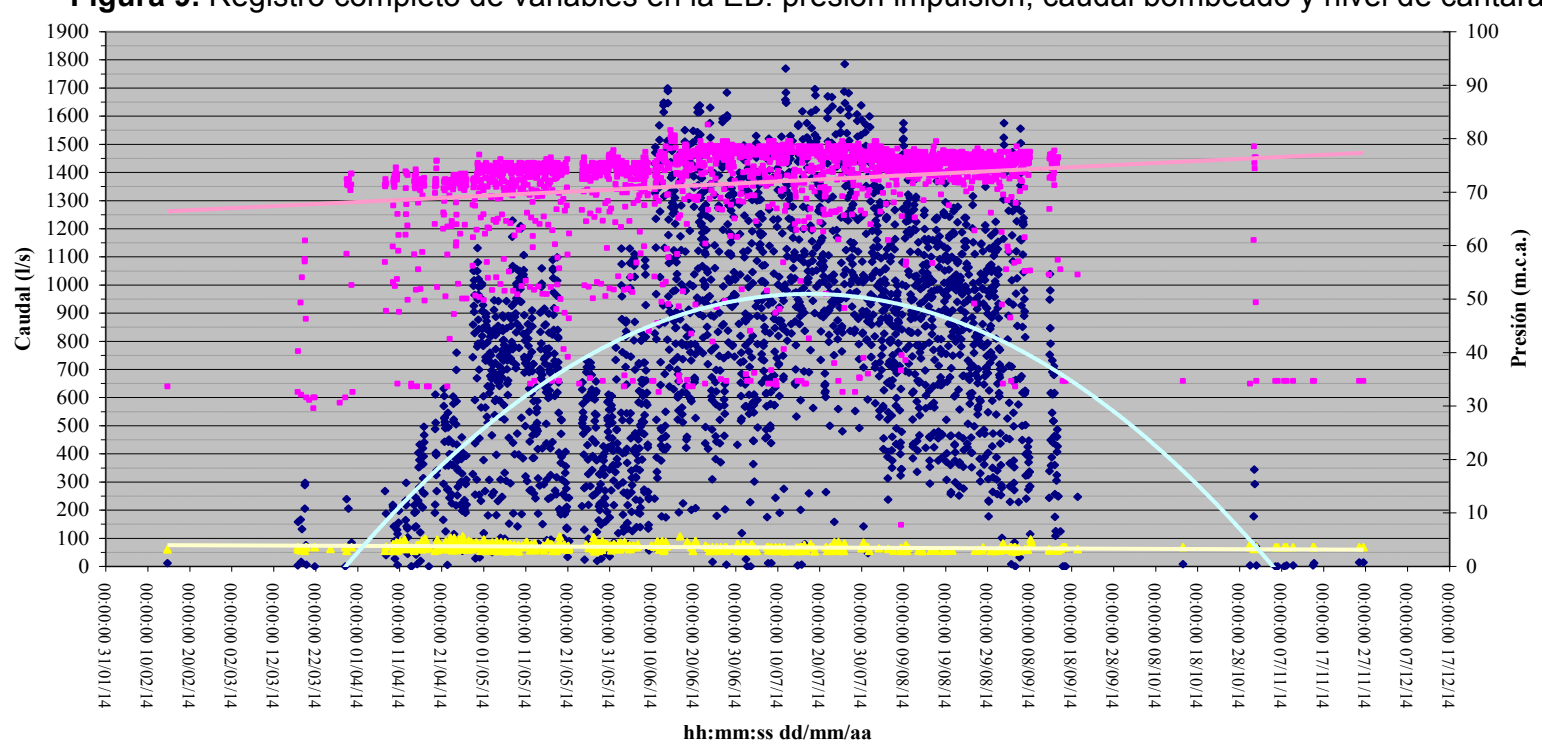

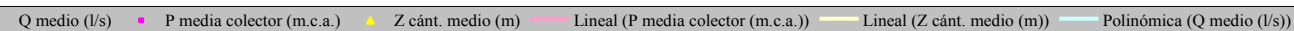

Para obtener el dato de la altura manométrica suministrada por los grupos de bombeo, a la presión registrada en el colector de impulsión habría que restarle la carga de agua sobre el rodete de la bomba en la cántara y sumarle la diferencia de altura entre el rodete de la bomba de eje vertical y la cota del transductor del colector de impulsión. 
Figura 10. Registro completo de variables en la estación de bombeo con altura manométrica de las bombas

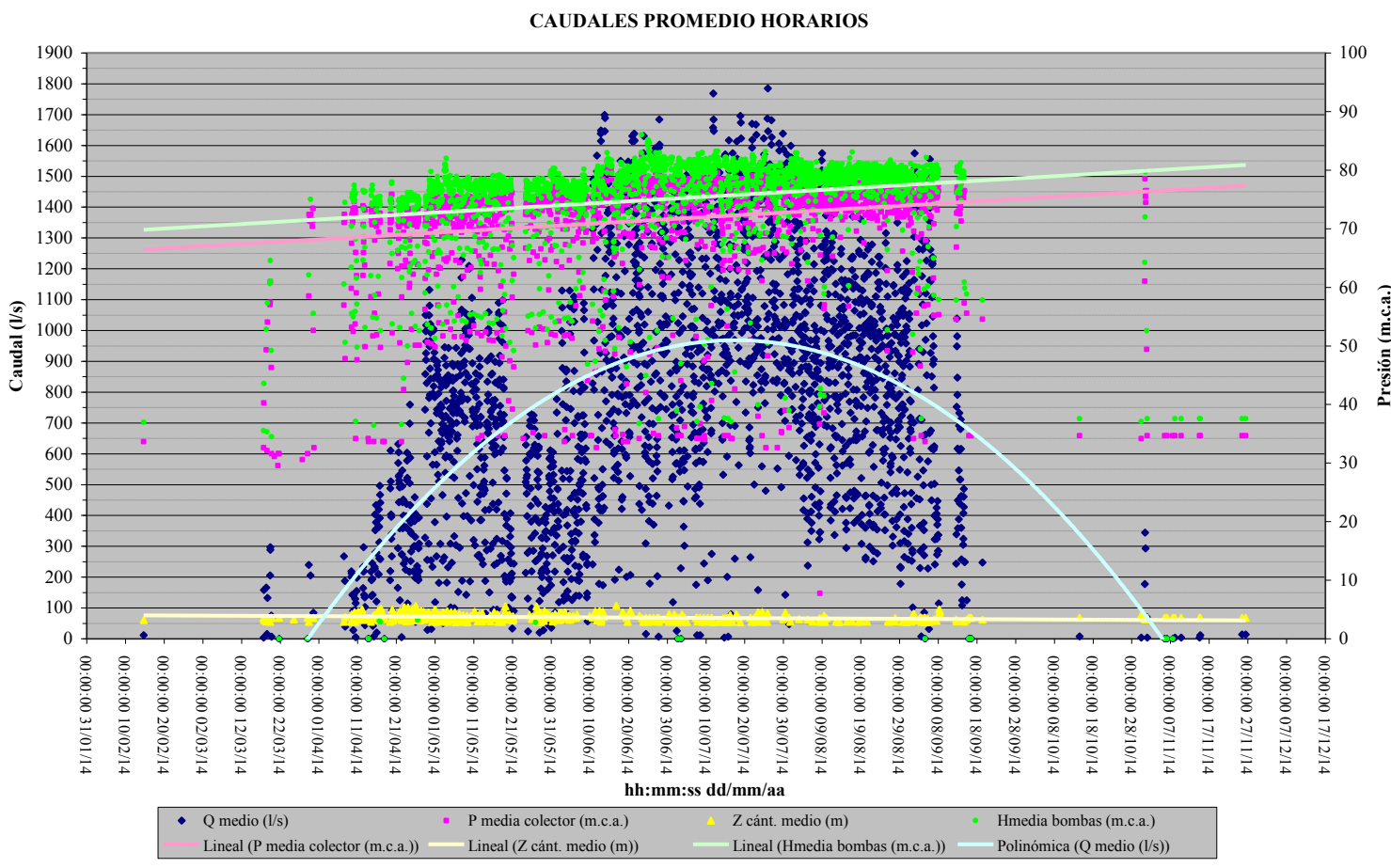

Para comprobar el régimen de funcionamiento de la EB se representa el gráfico de la altura manométrica de las bombas en función del caudal bombeado. También se representa la CCR teórica de la red, cuya expresión cuadrática viene dada por $\mathrm{H}(\mathrm{mca})=35+$ $0,0000127 \cdot \mathrm{Q}^{2}(\mathrm{I} / \mathrm{s}) ; \quad \mathrm{H}(\mathrm{mca})=35+12,654 \cdot \mathrm{Q}^{2}\left(\mathrm{~m}^{3} / \mathrm{s}\right)$.

Figura 11. Nube de puntos $H-Q$ de la EB y CCR

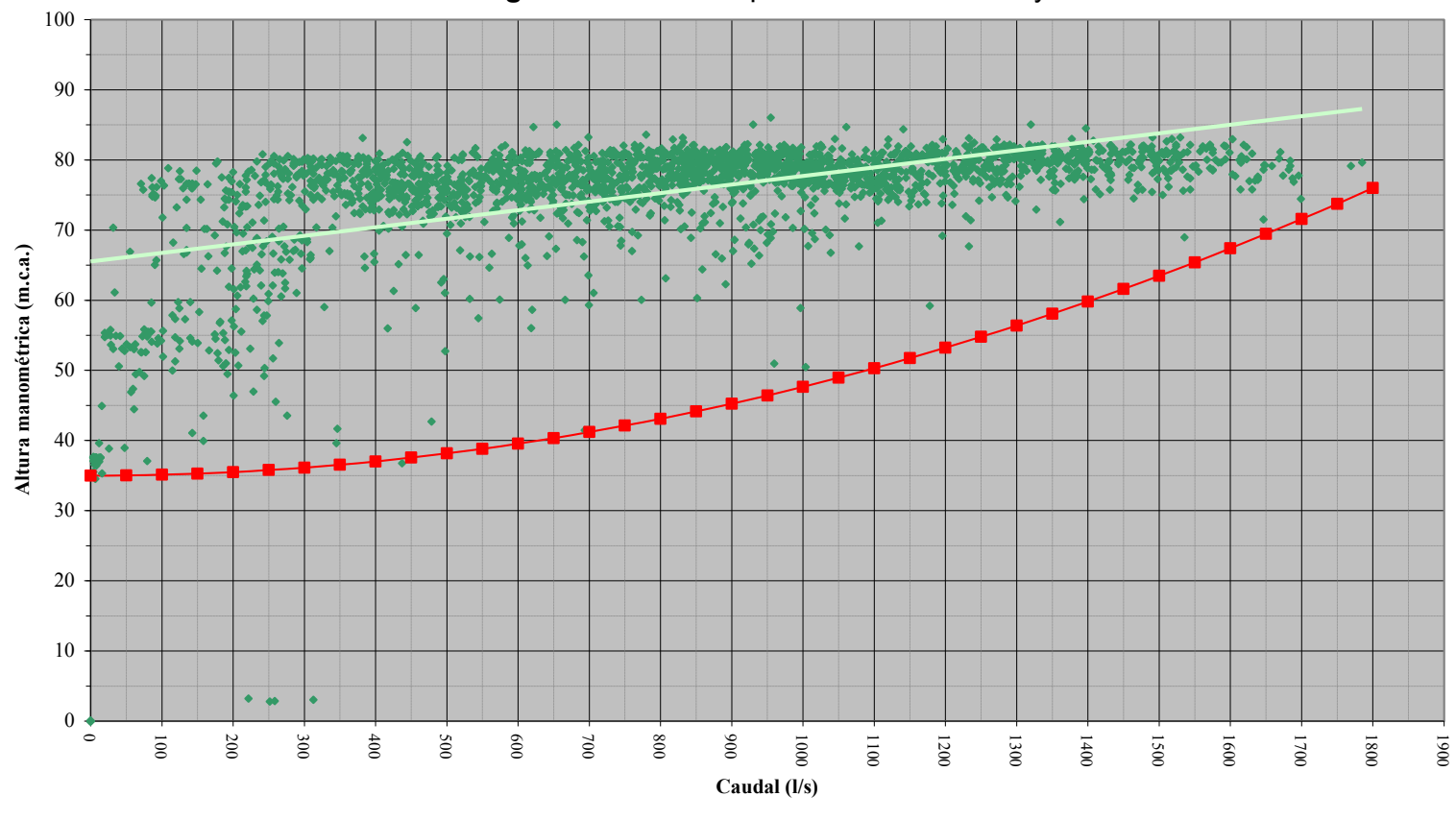

- Nube de punto Q-H $\rightarrow$ - Curva de consigna $\longrightarrow$ Tendencia Hmanométrica bombas

Se observa que la presión de bombeo a la que el SCADA está programado a punto 
fijo se encuentra por encima de la CCR teórica, por lo que el margen de mejora es amplio.

\section{8- Conclusiones y recomendaciones}

- El conocimiento del estado del arte ha permitido confeccionar en un único documento un recopilatorio completo de medidas de eficiencia energética cuya síntesis y redacción de las mismas se ha estructurado en varios bloques: equipos de bombeo, regulación de la $E B$, equipamiento instalaciones colectivas, equipamiento en instalaciones de parcela, consumo de agua, gestión, toma de datos y mediciones, formación y concienciación, y contratación del suministro eléctrico.

- El estudio de varias CC.RR. de CyL mediante técnicas de benchmarking han permitido caracterizar una parte del regadío modernizado castellanoleonés. Los bombeos se realizan desde captaciones superficiales tanto a balsa como a inyección directa red con un valor promedio del ICE de $76 \pm 17$ mca en los que predomina una regulación a presión constante, por lo que existe un alto margen de mejora para mejorar la eficiencia energética. El indicador hídrico de consumo de agua por unidad de área regada ( $\mathrm{VsSr}$ ) obtiene un valor promedio de $4.173 \pm 1.603 \mathrm{~m}^{3} \cdot \mathrm{ha}^{-1}$. La energía activa total consumida por unidad de área regada (EaSr) es de $1.403 \pm 497 \mathrm{kWh} \cdot \mathrm{ha}^{-1} \mathrm{y}$ por volumen de agua que entra en el sistema $\left(E a V_{T}\right)$ de $0,325 \pm 0,078 \mathrm{kWh} \cdot \mathrm{m}^{-3}$. La eficiencia energética de bombeo (EEB) se sitúa en un valor promedio del $66 \pm 13 \%$. El porcentaje de la factura eléctrica total que se destina al pago de la energía consumida (GCep) es del $71 \pm 13 \%$, y de la potencia contratada (GCep) del $28 \pm 13 \%$. El coste energético por área regada (CENSr) es de $140 \pm 58 € \cdot$ ha $^{-1}$ y por volumen suministrado (CENVs) de $0,035 \pm 0,013 € \cdot \mathrm{m}^{-3}$.

- La modelización informática de una infraestructura completa de riego colectivo con objeto de mejorar la eficiencia energética global del sistema conlleva a no separar la EB de la red de riego, y a analizar las sinergias que se producen entre ambas entidades. La documentación de partida para crear el modelo hidráulico así como el proceso de calibración llevado a cabo sobre el mismo es fundamental para el fin perseguido.

- Se debe de mejorar el protocolo de captación/almacenamiento de datos hidráulicos y eléctricos, así como revisar la calibración de las sondas y elementos de medición.

- En general, existe margen de mejora de la eficiencia energética trabajando en varios aspectos: ajuste de la potencia contratada; cambios en la organización del riego para aprovechar todas las horas baratas de los periodos tarifarios; afine de la regulación de los bombeos a las necesidades reales de la red (conocimiento de la curva motriz de la EB vs $\mathrm{CCR}$, entrada/salida y estados de transición de bombas en ciertos caudales); explotación de los datos históricos almacenados en el SCADA, el telecontrol y PROGAR; seguimiento de índices energéticos y comparativa con campañas anteriores; modelización de la red y EB para analizar estrategias de gestión (simulación predictiva de un patrón de riegos, reorganización de peticiones de riego mediante algoritmo de optimización de tipo evolutivo, supervisión del estado de la red...).

- La aplicación de las metodologías diseñadas en las zonas piloto, pone de manifiesto que realizar una buena gestión y mantenimiento es fundamental para conseguir que los regadíos sean eficientes desde el punto de vista hídrico y energético. Se trabaja aprovechando la tecnología disponible y creando una nueva herramienta para facilitar el intercambio de datos entre PROGAR y Telegestar.

- Desde el proyecto I+D+i OPTIREG que está llevando a cabo el grupo Tragsa se invita a las CC.RR. interesadas en mejorar su eficiencia energética a que participen de forma activa mediante un convenio de colaboración. 